SUPPLEMENTARY INFORMATION

\title{
An optimization and evaluation strategy of esophageal tissue preparation protocols for metabolomics by LC-MS
}

Huiqing Wang ${ }^{\dagger}$, Jing $\mathrm{Xu}^{\dagger}$, Yanhua Chen ${ }^{\dagger}$, Ruiping Zhang ${ }^{\dagger}$, Jiuming $\mathrm{He}^{\dagger}$, Zhonghua Wang ${ }^{\dagger}$, Qingce Zang ${ }^{\dagger}$, Jinfeng $\mathrm{We}^{\ddagger}$, Xiaowei Song ${ }^{\dagger}$, and Zeper Abliz, ${ }^{*}$

$\uparrow$ State Key Laboratory of Bioactive Substance and Function of Natural Medicines, Institute of Materia Medica, Chinese Academy of Medical Sciences and Peking Union Medical College, Beijing 100050, People's Republic of China

†New Drug Safety Evaluation Center, Institute of Materia Medica, Peking Union Medical College, Beijing 100050, People's Republic of China 


\section{CONTENTS}

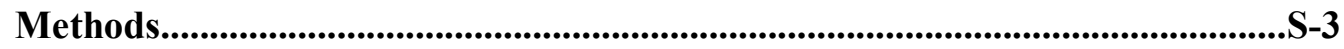

Supplementary Tables and Figures.........................................................................S-9

Table S1. Endogenous metabolites used to evaluate different methods....................S-9

Table S2. Method sensitivity............................................................................................5-15

Table S3. Instrument precision....................................................................................5-16

Table S4. Method precision..............................................................................................5-17

Table S5. Extraction recoveries of endogenous metabolites......................................S-18

Table S6. Freeze-thaw stability.......................................................................................

Figure S1. Typical TICs obtained from esophagus tissue by LC-(+)ESI-MS. A:

organic extracts; B: aqueous extracts...........................................................................2-21

Figure S2. Comparison of extraction efficiency using different solvent extraction methods for cholines (A), fatty acids (B), carnitines (C), and amino acids (D) in nonpolar extracts. Data are shown as column charts.................................................S-21

Figure S3. Comparison of extraction efficiency using different solvent extraction methods for amino acids (A), nucleosides (B), carnitines (C), and cholines (D) in polar extracts. Data are shown as column charts........................................................S-22

Figure S4. Comparison of extraction efficiency using different solvent extraction methods for positive and negative ion modes of ESI in nonpolar extracts (A, B) and polar extracts $(\mathrm{C}, \mathrm{D})$................................................................................................

Figure S5. Comparison of method reproducibility, evaluated by OPLS-DA (A) and error bars (B), for positive ion mode of ESI in nonpolar extracts...............................S-23

Figure S6. Peak features under different parameters in different extracts.................S-23

Figure S7. Workflow for esophageal tissue preparation..............................................S-24

Supplementary References..............................................................................................2-24 


\section{METHODS}

Sample collection. The metabolism study was approved by the Animal Care \& Welfare Committee, Institute of Materia Medica, Chinese Academy of Medical Sciences and Peking Union Medical College, and esophageal tissue was collected from the New Drug Safety Evaluation Center, Institute of Materia Medica, Peking Union Medical College. The esophageal tissue was washed with saline after surgical resection to avoid the impact of blood, placed directly into liquid nitrogen, and stored at $-80^{\circ} \mathrm{C}$ prior to analysis. Before metabolite extraction, esophageal tissue was cut into small pieces before homogenization to avoid heterogeneity homology.

Chemicals and Reagents. HPLC-grade acetonitrile (ACN) and formic acid were purchased from Merck (Darmstadt, Germany), and other chemicals were of analytical grade. Pure water was obtained from the Wahaha Group Co., Ltd., Hangzhou, China. L-tyrosine, proline, valine, phenylalanine, tryptophan, kynurenic acid, arachidonic acid, nicotinic acid, hippuric acid, linoleic acid, oleic acid, L-carnitine, lysoPC 16:0, acetylcarnitine, octanoylcarnitine, decanoylcarnitine, cytidine, inosine, and methyltestosterone were purchased from Sigma-Aldrich (St. Louis, MO, USA).

Sample Preparation. Methods for homogenization and metabolite extraction were as follows.

Three tissue disruption methods were used: $a . H W$ (Homogenized Wet): $50 \mathrm{mg}$ of wet tissue was accurately weighed, cut into small pieces, and extracted. $b . G W$ (Ground Wet): tissue was cut into pieces, ground in a liquid $\mathrm{N}_{2}$-cooled mortar and pestle, and placed into the freeze dryer to be lyophilized. Ten milligrams of accurately weighed lyophilized powder was then extracted (the weight of lyophilized tissue was $1 / 5$ that of wet tissue). c. $G D$ (Ground Dry): the opposite of $b . G W$; the sample was lyophilized first, and then ground into powder.

Four kinds of solvent extraction methods were used. Method 1 comprised methanol/water, dichloromethane/methanol (TS: two-step addition): corresponding tissue samples were accurately weighed into a $2 \mathrm{~mL}$ homogenization tube containing four ceramic beads ( $3.0 \mathrm{~mm}$ diameter). To the tube, $1.5 \mathrm{~mL}$ of pre-cooled extraction solvent (1:1 methanol/water) was added, and the tissue was homogenized three times for $30 \mathrm{~s}$ at a shock velocity of $4.0 \mathrm{~m} / \mathrm{s}$ using a high-throughput MasterPrep ${ }^{\mathrm{TM}}-24$ tissue homogenizer; the $30 \mathrm{~s}$ intervals were used to ensure maintenance of freezing temperatures in sample vials between homogenization steps. Homogenate was centrifuged at $15,000 \mathrm{rpm}$ for $5 \mathrm{~min}$ at $4^{\circ} \mathrm{C}$, and then $1.6 \mathrm{~mL}$ of pre-cooled $3: 1$ dichloromethane/methanol was added to the solid precipitate at lower homogenate after centrifuge, followed by another round of homogenization using the same parameters described above. The supernatants were collected and dried using a Savant SPD121P-230 SpeedVac concentrator with RVT-4104 refrigerated vapor traps (Thermo Fisher Scientific Electron Corporation, Milford, MA, USA). Dried polar and nonpolar extracts were stored at $-80^{\circ} \mathrm{C}$ prior to LC-MS analysis.

Method 2: dichloromethane/methanol/water ( $S W$ : stepwise addition) was the optimized protocol described in the article.

Method 3: dichloromethane/methanol/water ( $A O$ : all-in one addition) was similar to method 2. In this method, $1.5 \mathrm{~mL}$ of pre-cooled extraction solvent $(2: 2: 1.8$ 
dichloromethane/methanol/water) was added at one time. The rest of the operation was the same as in method 2.

Method 4: methanol/water (4:1, SP: single phase); $1.5 \mathrm{~mL}$ of pre-cooled extraction solvent (4:1 methanol/water) was added at one time, and the rest of the operation was the same as in method 3 .

The optimal protocol is detailed in the following steps. 1). Frozen tissue (wet weight: $50 \mathrm{mg}$ ) was accurately weighed into a $2 \mathrm{~mL}$ homogenization tube containing four ceramic beads (3.0 mm diameter). 2). Pre-cooled extraction solvents (410 $\mu \mathrm{L}$ of methanol, $210 \mu \mathrm{L}$ of water) were added, and the tissue was homogenized three times for $30 \mathrm{~s}$ at a shock velocity of $4.0 \mathrm{~m} / \mathrm{s}$ using a high-throughput MasterPrep ${ }^{\mathrm{TM}}-24$ tissue homogenizer. 3). After homogenization, $140 \mu \mathrm{L}$ of dichloromethane was added, and the sample was vortexed for $2 \mathrm{~min}$. 4). Then $140 \mu \mathrm{L}$ of dichloromethane and 210 $\mu \mathrm{L}$ of water were added, and the sample was vortexed for $2 \mathrm{~min}$ followed by incubation at $4^{\circ} \mathrm{C}$ for $20 \mathrm{~min}$. 5). Next, the sample was centrifuged at 15,000 rpm for 4 min at $4^{\circ}$ C. Centrifugation produced a biphasic mixture. 6). The upper (polar) and lower (nonpolar) layers were collected separately and dried using a Savant SPD121P-230 SpeedVac concentrator with RVT-4104 refrigerated vapor traps. 7). Before analysis, polar extracts were resuspended in 1.2 $\mathrm{mL}$ of 80:20 ACN/water, and nonpolar extracts were resuspended in $120 \mu \mathrm{L}$ of 40:60 ACN/water.

HPLC-MS conditions. Separation of analytes from the tissue extract was carried out using a Dionex UltiMate 3000 HPLC system, which consisted of an UltiMate 3000 RS pump, an UltiMate 3000 RS auto-sampler, and an UltiMate 3000 RS column compartment (Dionex, Olten, Switzerland). Gradient elution was performed as follows:

(1) HILIC-LC-MS analysis of polar extracts. A Phenomenex Kinetex HILIC column $(2.6 \mu \mathrm{m}, 2.1 \times 150 \mathrm{~mm})$ was used. Column temperature was $45^{\circ} \mathrm{C}$, and the flow rate was $0.3 \mathrm{~mL} / \mathrm{min}$. Mobile phase A was ACN and mobile phase B was $5 \mathrm{mM}$ ammonium acetate in water. Injection volumes of $10 \mu \mathrm{L}$ and $20 \mu \mathrm{L}$ were used for positive and negative ionization modes, respectively. The auto-sampler was set at $4^{\circ} \mathrm{C}$. The elution gradient was as follows:

\begin{tabular}{ccc}
\hline Retention Time(min) & A\% & B\% \\
\hline 0 & 95 & 5 \\
25 & 60 & 40 \\
30 & 95 & 5
\end{tabular}

(2) RP-LC-MS analysis of nonpolar extracts. A Waters ACQUITY UPLC CSH $\mathrm{C}_{18}$ column $(1.7 \mu \mathrm{m}, 2.1 \times 100 \mathrm{~mm})$ was used. Column temperature was $30^{\circ} \mathrm{C}$, and the flow rate was $0.2 \mathrm{~mL} / \mathrm{min}$. Mobile phase $\mathrm{A}$ was $\mathrm{ACN}$ and mobile phase $\mathrm{B}$ was $0.1 \%$ formic acid in water. An injection volume of $10 \mu \mathrm{L}$ was used for both positive and negative electrospray ionization (ESI) modes. The auto-sampler was set at $4^{\circ} \mathrm{C}$. The elution gradient was set as follows: 


\begin{tabular}{ccc}
\hline Retention Time $(\mathrm{min})$ & A\% & B\% \\
\hline 0 & 2 & 98 \\
3 & 60 & 40 \\
10 & 60 & 40 \\
12 & 81 & 19 \\
20 & 81 & 19 \\
22 & 90 & 10 \\
32 & 90 & 10 \\
33 & 100 & 0 \\
35 & 100 & 0 \\
\hline
\end{tabular}

The HPLC was coupled to a Q-Orbitrap mass spectrometer (Q Exactive, Thermo Fisher Scientific) via a heated electrospray source (HESI) using the following operation parameters: sheath gas flow rate, 40 PSI; aux gas flow rate, 11 Arb; sweep gas flow rate, 0 ; spray voltage, $3.5 \mathrm{kV}$ for positive mode and $3.2 \mathrm{kV}$ for negative mode; capillary temperature, $350^{\circ} \mathrm{C}$; s-lens RF level, 55; aux gas heater temperature, $220^{\circ} \mathrm{C}$. High-purity nitrogen $\left(\mathrm{N}_{2}\right)$ was used as the nebulizing gas and the collision gas for higher energy collisional dissociation.

LC-MS raw data files were converted to mzXML format using the Mass Matrix MS Data File Conversion Tools (http://www.massmatrix.net/). Peak finding, filtering, alignment, and scaling were performed using the open-source XCMS software (http://masspec.Scripps.edu/xcms/xcms.php) operated within the $\mathrm{R}$ statistical environment (version 2.15.2). The detailed parameters were as follows.

\section{Data Preprocessing.}

The program used for peak discrimination, filtering, alignment and CAMERA analysis of the LC-(+)ESI-MS data:

$\mathrm{rm}($ list $=1 \mathrm{~s}(\mathrm{all}=\mathrm{TRUE}))$

library(Biobase)

library(xcms)

library(multtest)

library(CAMERA)

sessionInfo()

$\mathrm{xs}<-\mathrm{xcmsSet}$ (profmethod="binlin",method="centWave",ppm=2.5, peakwidth $=\mathrm{c}(5,35)$, snthresh $=30$, prefilter $=c(10,10000)$, integrate $=1$, mzdiff $=0.005)$

xs $<$-group $(x s, b w=2$, minfrac $=0.3$, mzwid $=0.015)$

save(xs,file="xs.Rda")

ret.xs.obiwarp <-retcor(xs,method="obiwarp",plottype="deviation")

ret.xs.obiwarp<-group(ret.xs.obiwarp, bw $=2$, minfrac $=0.3$, mzwid $=0.015$ )

ret.xs.obiwarp

fill.ret.xs.obiwarp<-fillPeaks(ret.xs.obiwarp)

fill.ret.xs.obiwarp

save(fill.ret.xs.obiwarp, file="fill.ret.xs.obiwarp.Rda")

an. $1<$-annotate(fill.ret.xs.obiwarp,sigma $=6$,perfwhm $=0.3$,cor_eic_th $=0.75$,maxcharge $=3$, maxiso $=3$,mzabs $=0.03$, multiplier $=3$, polarity $=$ "positive")

peaklist.1<-getPeaklist(an.1) 
write.csv(peaklist.1,file='annotated.1.csv')

an. $2<$-annotate(fill.ret.xs.obiwarp,sigma $=6$,perfwhm $=0.3$, cor_eic_th $=0.75$, maxcharge $=3$, maxiso $=3$, mzabs $=0.03$, multiplier $=3$, polarity $=$ "positive")

peaklist. $2<$-getPeaklist(an.2)

write.csv(peaklist.2,file='annotated.2.csv')

an. $3<$-annotate(fill.ret.xs.obiwarp,sigma $=6$,perfwhm $=0.3$,cor_eic_th $=0.75$, maxcharge $=3$, maxiso $=3$, mzabs $=0.03$, multiplier $=3$, polarity $=$ "positive")

peaklist.3<-getPeaklist(an.3)

write.csv(peaklist.3,file='annotated.3.csv')

an. $4<$-annotate(fill.ret.xs.obiwarp,sigma $=6$,perfwhm $=0.3$,cor_eic_th $=0.75$, maxcharge $=3$, maxiso $=3$, mzabs $=0.03$, multiplier $=3$, polarity $=$ "positive")

peaklist.4<-getPeaklist(an.4)

write.csv(peaklist.4,file='annotated.4.csv')

report.fill.ret.xs.obiwarp<-diffreport(fill.ret.xs.obiwarp,"1","2",eicmax=8000,file="shi guan pos12")

save(report.fill.ret.xs.obiwarp,file="report.fill.ret.xs.obiwarp.Rad")

report.fill.ret.xs.obiwarp<-diffreport(fill.ret.xs.obiwarp,"1","3",eicmax=8000,file="shi guan pos13")

save(report.fill.ret.xs.obiwarp,file="report.fill.ret.xs.obiwarp.Rad")

report.fill.ret.xs.obiwarp<-diffreport(fill.ret.xs.obiwarp,"1","4",eicmax=8000,file="shi guan pos14")

save(report.fill.ret.xs.obiwarp,file="report.fill.ret.xs.obiwarp.Rad")

report.fill.ret.xs.obiwarp<-diffreport(fill.ret.xs.obiwarp,"2","3",eicmax=8000,file="shi guan pos23")

save(report.fill.ret.xs.obiwarp,file="report.fill.ret.xs.obiwarp.Rad")

report.fill.ret.xs.obiwarp<-diffreport(fill.ret.xs.obiwarp,"2","4",eicmax=8000,file="shi guan pos24")

save(report.fill.ret.xs.obiwarp,file="report.fill.ret.xs.obiwarp.Rad")

report.fill.ret.xs.obiwarp<-diffreport(fill.ret.xs.obiwarp,"3","4",eicmax=8000,file="shi guan pos34")

save(report.fill.ret.xs.obiwarp,file="report.fill.ret.xs.obiwarp.Rad")

The program used for the LC-(-)ESI-MS data:

rm(list=ls(all=TRUE) $)$

library(Biobase)

library(xcms)

library(multtest)

library(CAMERA)

sessionInfo()

$\mathrm{xs}<-\mathrm{xcmsSet}$ (profmethod="binlin",method="centWave",ppm=2.5, peakwidth $=\mathrm{c}(5,35)$, snthresh $=30$, prefilter $=c(10,10000)$,integrate $=1$, mzdiff $=0.005)$

$\mathrm{xs}<$-group $(\mathrm{xs}, \mathrm{bw}=2, \operatorname{minfrac}=0.3, \mathrm{mzwid}=0.015)$

save(xs,file="xs.Rda")

ret.xs.obiwarp <-retcor(xs,method="obiwarp",plottype="deviation")

ret.xs.obiwarp $<$-group(ret.xs.obiwarp, $b w=2, \operatorname{minfrac}=0.3, \mathrm{mzwid}=0.015$ ) 
ret.xs.obiwarp

fill.ret.xs.obiwarp<-fillPeaks(ret.xs.obiwarp)

fill.ret.xs.obiwarp

save(fill.ret.xs.obiwarp, file="fill.ret.xs.obiwarp.Rda")

an. $1<$-annotate(fill.ret.xs.obiwarp,sigma $=6$,perfwhm $=0.3$, cor_eic_th $=0.75$, maxcharge

$=3$, maxiso $=3$, mzabs $=0.03$, multiplier $=3$, polarity $=$ "negative")

peaklist. $1<$-getPeaklist(an.1)

write.csv(peaklist.1,file='annotated.1.csv')

an. $2<$-annotate(fill.ret.xs.obiwarp,sigma $=6$,perfwhm $=0.3$,cor_eic_th $=0.75$, maxcharge

$=3$, maxiso $=3$, mzabs $=0.03$, multiplier $=3$, polarity $=$ "negative")

peaklist. $2<$-getPeaklist(an.2)

write.csv(peaklist.2, file='annotated.2.csv')

an. $3<$-annotate(fill.ret.xs.obiwarp,sigma $=6$,perfwhm $=0.3$,cor_eic_th $=0.75$, maxcharge

$=3$, maxiso $=3$, mzabs $=0.03$, multiplier $=3$, polarity $=$ "negative")

peaklist.3<-getPeaklist(an.3)

write.csv(peaklist.3,file='annotated.3.csv')

an. $4<$-annotate(fill.ret.xs.obiwarp,sigma $=6$,perfwhm $=0.3$,cor_eic_th $=0.75$, maxcharge

$=3$, maxiso $=3$, mzabs $=0.03$, multiplier $=3$, polarity $=$ "negative")

peaklist.4<-getPeaklist(an.4)

write.csv(peaklist.4,file='annotated.4.csv')

report.fill.ret.xs.obiwarp<-diffreport(fill.ret.xs.obiwarp,"1","2",eicmax=8000,file="shi guan neg12")

save(report.fill.ret.xs.obiwarp,file="report.fill.ret.xs.obiwarp.Rad")

report.fill.ret.xs.obiwarp<-diffreport(fill.ret.xs.obiwarp,"1","3",eicmax=8000,file="shi guan neg13")

save(report.fill.ret.xs.obiwarp,file="report.fill.ret.xs.obiwarp.Rad")

report.fill.ret.xs.obiwarp<-diffreport(fill.ret.xs.obiwarp,"1","4",eicmax=8000,file="shi guan neg14")

save(report.fill.ret.xs.obiwarp,file="report.fill.ret.xs.obiwarp.Rad")

report.fill.ret.xs.obiwarp<-diffreport(fill.ret.xs.obiwarp,"2","3",eicmax=8000,file="shi

guan neg23")

save(report.fill.ret.xs.obiwarp,file="report.fill.ret.xs.obiwarp.Rad")

report.fill.ret.xs.obiwarp<-diffreport(fill.ret.xs.obiwarp,"2","4",eicmax=8000,file="shi

guan neg24")

save(report.fill.ret.xs.obiwarp,file="report.fill.ret.xs.obiwarp.Rad")

report.fill.ret.xs.obiwarp<-diffreport(fill.ret.xs.obiwarp,"3","4",eicmax=8000,file="shi guan neg34")

save(report.fill.ret.xs.obiwarp,file="report.fill.ret.xs.obiwarp.Rad")

The resultant two-dimensional matrices, including observations (sample names) in columns, variables ( $\mathrm{m} / \mathrm{z}$-retention time pairs) in rows, and peak areas, were further imported into the SIMCA-P 12.0 software package (Umetrics AB, Umeå, Sweden) for multivariate statistical data analysis (MVDA). Mean-centering and Pareto-scaling were applied to all data prior to MVDA to reduce noise and artifacts in the models. Orthogonal partial least-squares discriminant analysis (OPLS-DA) was used to 
evaluate the reproducibility of different methods. To test the validity of the model against overfitting, the cross-validation parameter Q2 was computed as in partial least-squares discriminant analysis (PLS-DA) by permutation testing using 100 random permutations.

Method validation. For preparation of endogenous standard mixture solution (MIX), the following 19 endogenous standards were accurately weighed (5 mg each) into a $1 \mathrm{~mL}$ tube (containing methanol or methanol/water as a solvent): L-tyrosine, proline, valine, phenylalanine, tryptophan, kynurenic acid, arachidonic acid, nicotinic acid, hippuric acid, linoleic acid, oleic acid, L-carnitine, lysoPC 16:0, acetylcarnitine, octanoylcarnitine, decanoylcarnitine, cytidine, inosine, and methyltestosterone. The standard solutions were saved as stock solutions. Different volumes of these standard solutions were collected, mixed together, and diluted into $2 \mathrm{~mL}$ of water, followed by vortexing for 30 seconds to ensure complete mixing. The concentration of each analyte in $M I X$ was 10 times its concentration in normal tissues. This standard solution was used for the method validation study.

Sensitivity. The MIX solution was separately diluted 1-, 2-, 5-, 10-, 50-, or 100 -fold, each with two replicates. Thus, the concentrations of analytes in the diluted solutions were $10,5,2,1,1 / 5$, and $1 / 10$ times their concentrations in normal tissues ${ }^{1}$ (concentrations in the normal mouse tissues can be found in database of MMDB); these solutions were labeled mix 1 , mix2, mix3, mix4, mix5, and mix6, respectively. Established LC-MS methods were used to calculate the minimum detectable concentration of each compound. When the actual minimum detectable concentration of the sample was less than $1 / 10^{\text {th }}$ of that in normal tissue, $1 / 10^{\text {th }}$ of the normal tissue concentration was defined as the minimum detectable concentration, and the signal-to-noise ratio $(\mathrm{S} / \mathrm{N})$ was recorded under that condition.

Precision. Instrument precision was characterized by relative standard deviation (RSD) of the extracted ion chromatogram peak area from six continuous injections of the same sample. Method precision: Intra-day precision was evaluated by freshly preparing six independent replicates $(n=6)$ of tissue samples; mix3 (2 times normal tissue concentration) was added into the tissues. The inter-day precision was monitored using six replicates of the samples described above on three different days. As shown in Table S3, the RSD of metabolite extracted peak area was 1.9-16.2\%. Results of the method precision study are shown in Table S4; the inter- and intra-day precisions were $4.4-23.2 \%$ and $3.5-20.9 \%$, respectively. These results indicate that our protocol is reproducible and reliable for esophageal tissue metabolomics research.

Recovery. mix 2, mix 3 , and mix4 $(5,2$, and 1 times the normal tissue concentration respectively) were added to the blank tissues using the tissue preparation protocol described above; these were defined as high, middle, and low concentration levels, respectively (replicates: $n=3$ ). Standard solutions at the corresponding concentrations were analyzed in parallel. Molecular recovery was calculated as ([area of spiked tissue samples - area of blank tissue samples]/[area of spiked after preparation tissue samples - area of blank tissue samples])*100\%. The extraction recoveries of the 19 compounds at three different concentrations are summarized in Table S5. Extraction recoveries were 50.7-128.6\%, and mean recovery 
was $89.6 \%$. Most of the compounds are present in both polar and nonpolar extracts, but the proportion differed according to the polarity. Recovery was the sum of the two parts. Thus, extraction recoveries of our method meet the acceptance criteria.

Stability. To study metabolite stability under analysis conditions, sets of samples were prepared and either incubated in the auto-sampler at $4^{\circ} \mathrm{C}$ for $1,2,3$, or 7 days, or subjected to three freeze-thaw cycles $\left(-20^{\circ} \mathrm{C}-20^{\circ} \mathrm{C}\right)$. Stability was characterized as $\mathrm{RSD}$ of the extracted ion chromatogram peak areas of endogenous standard compounds.

\section{SUPPLEMENTARY TABLES AND FIGURES}

Table S1. Endogenous metabolites used to evaluate different methods.

\begin{tabular}{|c|c|c|c|c|c|}
\hline Polar-Pos & No. & Compound & Formula & {$[\mathbf{M}+\mathbf{H}]^{+}$} & Retention Time \\
\hline & 1 & Valine $^{\text {a }}$ & $\mathrm{C}_{5} \mathrm{H}_{11} \mathrm{NO}_{2}$ & 118.0863 & 11.62 \\
\hline & 2 & L-isoleucine $^{\mathrm{a}}$ & $\mathrm{C}_{6} \mathrm{H}_{13} \mathrm{NO}_{2}$ & 132.1019 & 12.57 \\
\hline & 3 & L-leucine ${ }^{\text {a }}$ & $\mathrm{C}_{6} \mathrm{H}_{13} \mathrm{NO}_{2}$ & 132.1019 & 12.57 \\
\hline & 4 & L-methionine $^{\mathrm{a}}$ & $\mathrm{C}_{5} \mathrm{H}_{11} \mathrm{NO}_{2} \mathrm{~S}$ & 150.0583 & 11.41 \\
\hline & 5 & Phenylalanine $^{\mathrm{a}}$ & $\mathrm{C}_{9} \mathrm{H}_{11} \mathrm{NO}_{2}$ & 166.0863 & 10.99 \\
\hline & 6 & Proline ${ }^{a}$ & $\mathrm{C}_{5} \mathrm{H}_{9} \mathrm{NO}_{2}$ & 116.0706 & 13.69 \\
\hline & 7 & Hydroxy-L-proline ${ }^{a}$ & $\mathrm{C}_{5} \mathrm{H}_{9} \mathrm{NO}_{3}$ & 132.0655 & 13.44 \\
\hline Polar extracts & 8 & Tryptophan ${ }^{\text {a }}$ & $\mathrm{C}_{11} \mathrm{H}_{12} \mathrm{~N}_{2} \mathrm{O}_{2}$ & 205.0972 & 10.61 \\
\hline in positive ion mode of & 9 & L-Tyrosine ${ }^{a}$ & $\mathrm{C}_{9} \mathrm{H}_{11} \mathrm{NO}_{3}$ & 182.0812 & 12.78 \\
\hline ESI & 10 & L-glutamine ${ }^{a}$ & $\mathrm{C}_{5} \mathrm{H}_{10} \mathrm{~N}_{2} \mathrm{O}_{3}$ & 147.0764 & 17.38 \\
\hline \multirow[t]{12}{*}{ Amino Acids } & 11 & Phenylacetylglutamine ${ }^{\text {a }}$ & $\mathrm{C}_{13} \mathrm{H}_{16} \mathrm{~N}_{2} \mathrm{O}_{4}$ & 265.1183 & 6.79 \\
\hline & 12 & L-cystine $^{b}$ & $\mathrm{C}_{6} \mathrm{H}_{12} \mathrm{~N}_{2} \mathrm{O}_{4} \mathrm{~S}_{2}$ & 241.0311 & 4.03 \\
\hline & 13 & S-carboxymethylcysteine ${ }^{b}$ & $\mathrm{C}_{5} \mathrm{H}_{9} \mathrm{NO}_{4} \mathrm{~S}$ & 180.0325 & 2.96 \\
\hline & 14 & L-glutamic acid ${ }^{\text {a }}$ & $\mathrm{C}_{5} \mathrm{H}_{9} \mathrm{NO}_{4}$ & 148.0604 & 3.12 \\
\hline & 15 & Taurine $^{\mathrm{a}}$ & $\mathrm{C}_{2} \mathrm{H}_{7} \mathrm{NO}_{3} \mathrm{~S}$ & 126.0219 & 6.99 \\
\hline & 16 & L-citrulline $^{\text {a }}$ & $\mathrm{C}_{6} \mathrm{H}_{13} \mathrm{~N}_{3} \mathrm{O}_{3}$ & 176.103 & 15.92 \\
\hline & 17 & (R)-(+)-2-Pyrrolidone-5-carboxylic acid ${ }^{\text {a }}$ & $\mathrm{C}_{5} \mathrm{H}_{7} \mathrm{NO}_{3}$ & 130.0499 & 18.45 \\
\hline & 18 & Creatine $^{a}$ & $\mathrm{C}_{4} \mathrm{H}_{9} \mathrm{~N}_{3} \mathrm{O}_{2}$ & 132.0768 & 14.08 \\
\hline & 1 & L-Carnitine $^{\text {a }}$ & $\mathrm{C}_{7} \mathrm{H}_{15} \mathrm{NO}_{3}$ & 162.1125 & 19.56 \\
\hline & 2 & $\gamma$-butyrobetaine ${ }^{b}$ & $\mathrm{C}_{7} \mathrm{H}_{15} \mathrm{NO}_{2}$ & 146.1176 & 23.66 \\
\hline & 3 & L-Acetylcarnitine ${ }^{\text {a }}$ & $\mathrm{C}_{9} \mathrm{H}_{17} \mathrm{NO}_{4}$ & 204.123 & 17.92 \\
\hline & 4 & Hexanoylcarnitine(C6:0) ${ }^{\mathrm{b}}$ & $\mathrm{C}_{13} \mathrm{H}_{25} \mathrm{NO}_{4}$ & 260.1856 & 14.93 \\
\hline Polar extracts & 5 & Heptanoylcarnitine $(\mathrm{C} 7: 0)^{\mathrm{b}}$ & $\mathrm{C}_{14} \mathrm{H}_{27} \mathrm{NO}_{4}$ & 274.2013 & 17.71 \\
\hline in positive ion mode of & 6 & Octanoylcarnitine $(\mathrm{C} 8: 0)^{\mathrm{a}}$ & $\mathrm{C}_{15} \mathrm{H}_{29} \mathrm{NO}_{4}$ & 288.2169 & 13.98 \\
\hline ESI & 7 & Nonanoylcarnitine $(\mathrm{C} 9: 0)^{\mathrm{b}}$ & $\mathrm{C}_{16} \mathrm{H}_{31} \mathrm{NO}_{4}$ & 302.2326 & 13.60 \\
\hline \multirow[t]{5}{*}{ Carnitines } & 8 & Decanoylcarnitine $(\mathrm{C} 10: 0)^{\mathrm{b}}$ & $\mathrm{C}_{17} \mathrm{H}_{33} \mathrm{NO}_{4}$ & 316.2482 & 13.52 \\
\hline & 9 & Undecanoylcarnitine $(11: 0)^{\mathrm{b}}$ & $\mathrm{C}_{18} \mathrm{H}_{35} \mathrm{NO}_{4}$ & 330.2639 & 1.42 \\
\hline & 10 & Octenoylcarnitine $(\mathrm{C} 8: 1)^{\mathrm{b}}$ & $\mathrm{C}_{15} \mathrm{H}_{27} \mathrm{NO}_{4}$ & 286.2013 & 14.20 \\
\hline & 11 & Decenoylcarnitine $(\mathrm{C} 10: 1)^{\mathrm{b}}$ & $\mathrm{C}_{17} \mathrm{H}_{31} \mathrm{NO}_{4}$ & 314.2326 & 13.52 \\
\hline & 12 & Tetradecadiennoylcarnitine $(14: 2)^{\mathrm{b}}$ & $\mathrm{C}_{21} \mathrm{H}_{37} \mathrm{NO}_{4}$ & 368.2795 & 13.02 \\
\hline
\end{tabular}




\begin{tabular}{|c|c|c|c|c|c|}
\hline & No. & Compound & Formula & {$[\mathbf{M}+\mathbf{H}]^{+}$} & Retention Time \\
\hline & 13 & 3-OH-octanoylcarnitine $(\mathrm{C} 8: 0-\mathrm{OH})^{\mathrm{b}}$ & $\mathrm{C}_{15} \mathrm{H}_{29} \mathrm{NO}_{5}$ & 304.2118 & 15.23 \\
\hline & 14 & Hydroxydecanoylcarnitine $(\mathrm{C} 10: 0-\mathrm{OH})^{\mathrm{b}}$ & $\mathrm{C}_{17} \mathrm{H}_{33} \mathrm{NO}_{5}$ & 332.2432 & 14.96 \\
\hline & 15 & Carnitine $\mathrm{C} 12: 0-\mathrm{OH}^{\mathrm{b}}$ & $\mathrm{C}_{19} \mathrm{H}_{37} \mathrm{NO}_{5}$ & 360.2745 & 14.29 \\
\hline & 16 & Carnitine $\mathrm{C} 14: 0-\mathrm{OH}^{\mathrm{b}}$ & $\mathrm{C}_{21} \mathrm{H}_{41} \mathrm{NO}_{5}$ & 388.3058 & 13.74 \\
\hline & 17 & Carnitine $\mathrm{C} 14: 2-\mathrm{OH}^{\mathrm{b}}$ & $\mathrm{C}_{21} \mathrm{H}_{37} \mathrm{NO}_{5}$ & 384.2745 & 13.71 \\
\hline & 18 & Carnitine $\mathrm{C} 16: 2-\mathrm{OH}^{\mathrm{b}}$ & $\mathrm{C}_{23} \mathrm{H}_{41} \mathrm{NO}_{5}$ & 412.3058 & 13.58 \\
\hline & 19 & Carnitine $\mathrm{C} 18: 2-\mathrm{OH}^{\mathrm{b}}$ & $\mathrm{C}_{25} \mathrm{H}_{45} \mathrm{NO}_{5}$ & 440.3371 & 13.22 \\
\hline & 1 & $\operatorname{LPC}(14: 0)^{b}$ & $\mathrm{C}_{22} \mathrm{H}_{46} \mathrm{NO}_{7} \mathrm{P}$ & 468.3085 & 13.25 \\
\hline & 2 & $\operatorname{LPC}(15: 0)^{\mathrm{a}}$ & $\mathrm{C}_{23} \mathrm{H}_{48} \mathrm{NO}_{7} \mathrm{P}$ & 482.3241 & 13.29 \\
\hline & 3 & $\operatorname{LPC}(16: 0)^{\mathrm{a}}$ & $\mathrm{C}_{24} \mathrm{H}_{50} \mathrm{NO}_{7} \mathrm{P}$ & 496.3398 & 13.29 \\
\hline & 4 & $\operatorname{LPC}(17: 0)^{b}$ & $\mathrm{C}_{25} \mathrm{H}_{52} \mathrm{NO}_{7} \mathrm{P}$ & 510.3554 & 13.10 \\
\hline & 5 & $\operatorname{LPC}(18: 0)^{b}$ & $\mathrm{C}_{26} \mathrm{H}_{54} \mathrm{NO}_{7} \mathrm{P}$ & 524.3711 & 15.73 \\
\hline & 6 & $\operatorname{LPC}(21: 0)^{\mathrm{b}}$ & $\mathrm{C}_{30} \mathrm{H}_{50} \mathrm{NO}_{7} \mathrm{P}$ & 568.3398 & 11.71 \\
\hline Polar extracts & 7 & $\operatorname{LPC}(16: 1)^{b}$ & $\mathrm{C}_{24} \mathrm{H}_{48} \mathrm{NO}_{7} \mathrm{P}$ & 494.3241 & 16.1 \\
\hline in positive ion mode & 8 & $\operatorname{LPC}(17: 1)^{b}$ & $\mathrm{C}_{25} \mathrm{H}_{50} \mathrm{NO}_{7} \mathrm{P}$ & 508.3398 & 15.95 \\
\hline of ESI & 9 & $\operatorname{LPC}(18: 1)^{a}$ & $\mathrm{C}_{26} \mathrm{H}_{52} \mathrm{NO}_{7} \mathrm{P}$ & 522.3554 & 13.18 \\
\hline \multirow[t]{7}{*}{ Cholines } & 10 & $\operatorname{LPC}(18: 2)^{b}$ & $\mathrm{C}_{26} \mathrm{H}_{50} \mathrm{NO}_{7} \mathrm{P}$ & 520.3398 & 13.04 \\
\hline & 11 & $\operatorname{LPC}(18: 3)^{b}$ & $\mathrm{C}_{26} \mathrm{H}_{48} \mathrm{NO}_{7} \mathrm{P}$ & 518.3241 & 13.10 \\
\hline & 12 & $\operatorname{LPC}(20: 3)^{b}$ & $\mathrm{C}_{28} \mathrm{H}_{52} \mathrm{NO}_{7} \mathrm{P}$ & 546.3554 & 12.86 \\
\hline & 13 & $\operatorname{LPC}(20: 4)^{b}$ & $\mathrm{C}_{28} \mathrm{H}_{50} \mathrm{NO}_{7} \mathrm{P}$ & 544.3398 & 12.96 \\
\hline & 14 & $\operatorname{LPC}(20: 5)^{b}$ & $\mathrm{C}_{28} \mathrm{H}_{48} \mathrm{NO}_{7} \mathrm{P}$ & 542.3241 & 15.94 \\
\hline & 15 & $\operatorname{LPE}(22: 6)^{b}$ & $\mathrm{C}_{27} \mathrm{H}_{44} \mathrm{NO}_{7} \mathrm{P}$ & 526.2928 & 12.59 \\
\hline & 16 & $\operatorname{LPE}(20: 4)^{\mathrm{b}}$ & $\mathrm{C}_{25} \mathrm{H}_{44} \mathrm{NO}_{7} \mathrm{P}$ & 502.2928 & 12.82 \\
\hline Polar-POS & 1 & 1-methylguanine ${ }^{a}$ & $\mathrm{C}_{6} \mathrm{H}_{7} \mathrm{~N}_{5} \mathrm{O}$ & 166.0723 & 4.93 \\
\hline \multirow[t]{8}{*}{ Nucleosides } & 2 & Hypoxanthine ${ }^{a}$ & $\mathrm{C}_{5} \mathrm{H}_{4} \mathrm{~N}_{4} \mathrm{O}$ & 137.0458 & 3.61 \\
\hline & 3 & 3-Methylxanthine ${ }^{a}$ & $\mathrm{C}_{6} \mathrm{H}_{6} \mathrm{~N}_{4} \mathrm{O}_{2}$ & 167.0564 & 2.06 \\
\hline & 4 & 7-Methylxanthine ${ }^{a}$ & $\mathrm{C}_{6} \mathrm{H}_{6} \mathrm{~N}_{4} \mathrm{O}_{2}$ & 167.0564 & 5.95 \\
\hline & 5 & Dimethylguanosine ${ }^{c}$ & $\mathrm{C}_{12} \mathrm{H}_{17} \mathrm{~N}_{5} \mathrm{O}_{5}$ & 312.1302 & 13.06 \\
\hline & 6 & 5-Methylthioadenosine ${ }^{a}$ & $\mathrm{C}_{11} \mathrm{H}_{15} \mathrm{~N}_{5} \mathrm{O}_{3} \mathrm{~S}$ & 298.0968 & 2.75 \\
\hline & 7 & Cytidine $^{a}$ & $\mathrm{C}_{9} \mathrm{H}_{13} \mathrm{~N}_{3} \mathrm{O}_{5}$ & 244.0928 & 5.15 \\
\hline & 8 & $2^{\prime}$-Deoxycytidine ${ }^{a}$ & $\mathrm{C}_{9} \mathrm{H}_{13} \mathrm{~N}_{3} \mathrm{O}_{4}$ & 228.0979 & 5.44 \\
\hline & 9 & $\mathrm{cGMP}^{\mathrm{b}}$ & $\mathrm{C}_{10} \mathrm{H}_{12} \mathrm{~N}_{5} \mathrm{O}_{7} \mathrm{P}$ & 346.0547 & 5.85 \\
\hline \multirow{2}{*}{ Polar extracts } & 1 & Pregnenolone ${ }^{b}$ & $\mathrm{C}_{21} \mathrm{H}_{32} \mathrm{O}_{2}$ & 317.2475 & 4.06 \\
\hline & 2 & Androsterone sulfate ${ }^{b}$ & $\mathrm{C}_{19} \mathrm{H}_{30} \mathrm{O}_{5} \mathrm{~S}$ & 371.1887 & 1.83 \\
\hline \multirow[t]{2}{*}{ on mode of } & 3 & DHEA sulfate ${ }^{b}$ & $\mathrm{C}_{19} \mathrm{H}_{28} \mathrm{O}_{5} \mathrm{~S}$ & 369.173 & 1.96 \\
\hline & 4 & L-Fucose ${ }^{b}$ & $\mathrm{C}_{6} \mathrm{H}_{12} \mathrm{O}_{5}$ & 165.0758 & 1.90 \\
\hline Low-level metabolites & 5 & D-sorbitol ${ }^{\text {b }}$ & $\mathrm{C}_{6} \mathrm{H}_{14} \mathrm{O}_{6}$ & 183.0863 & 6.87 \\
\hline
\end{tabular}




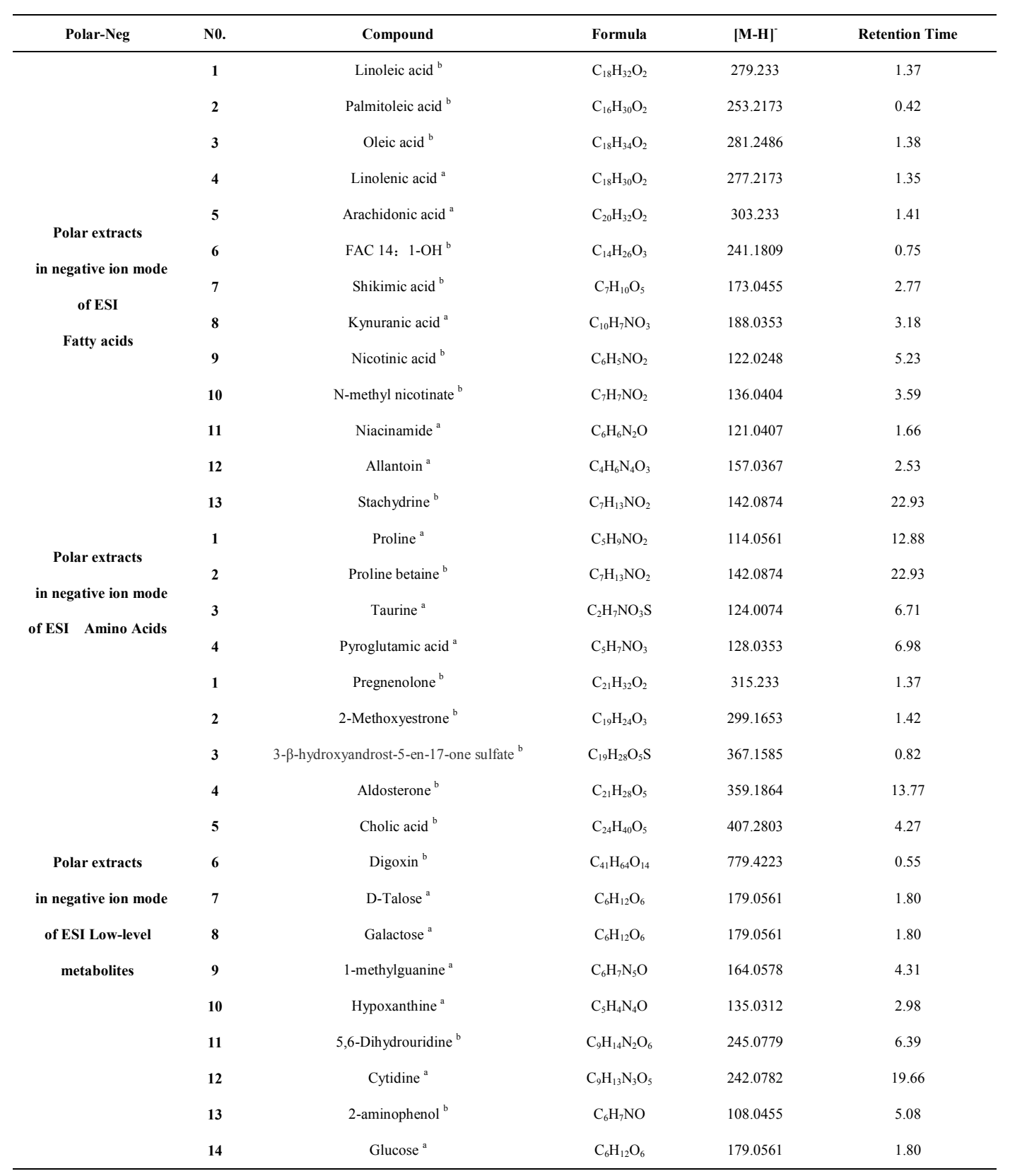




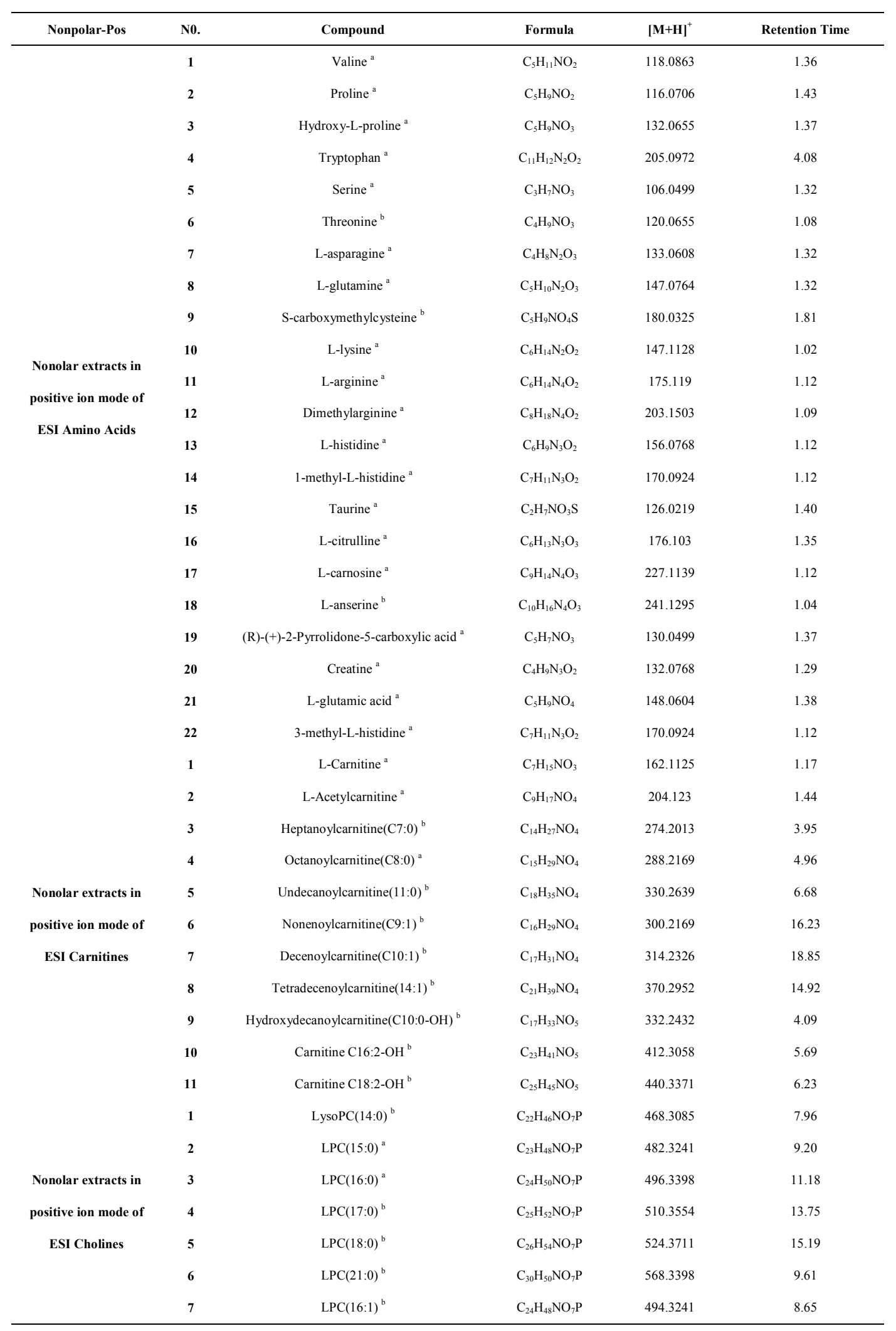




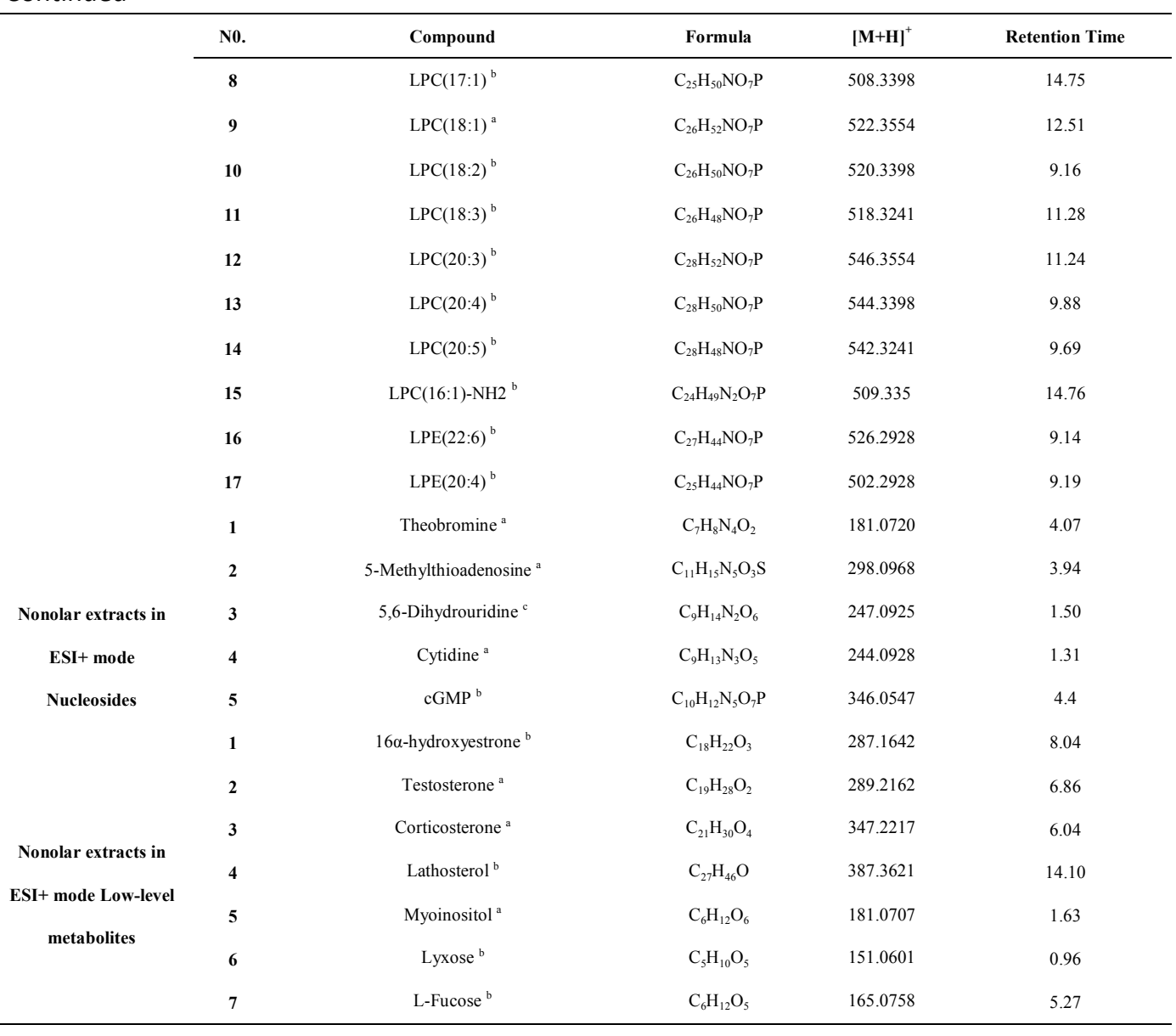




\begin{tabular}{|c|c|c|c|c|c|}
\hline Nonpolar-Neg & No. & Compound & Formula & {$[\mathbf{M - H}]^{-}$} & Retention Time \\
\hline \multirow[b]{7}{*}{ Nonolar extracts in } & 1 & L-isoleucine $^{\mathrm{a}}$ & $\mathrm{C}_{6} \mathrm{H}_{13} \mathrm{NO}_{2}$ & 130.0874 & 1.26 \\
\hline & 2 & Hydroxy-L-proline ${ }^{a}$ & $\mathrm{C}_{5} \mathrm{H}_{9} \mathrm{NO}_{3}$ & 130.051 & 1.38 \\
\hline & 3 & Serine $^{a}$ & $\mathrm{C}_{3} \mathrm{H}_{7} \mathrm{NO}_{3}$ & 104.0353 & 1.32 \\
\hline & 4 & Threonine $^{\text {a }}$ & $\mathrm{C}_{4} \mathrm{H}_{9} \mathrm{NO}_{3}$ & 118.051 & 1.34 \\
\hline & 5 & L-asparagine ${ }^{a}$ & $\mathrm{C}_{4} \mathrm{H}_{8} \mathrm{~N}_{2} \mathrm{O}_{3}$ & 131.0462 & 1.34 \\
\hline & 6 & L-glutamine ${ }^{a}$ & $\mathrm{C}_{5} \mathrm{H}_{10} \mathrm{~N}_{2} \mathrm{O}_{3}$ & 145.0619 & 1.34 \\
\hline & 7 & L-lysine $^{a}$ & $\mathrm{C}_{6} \mathrm{H}_{14} \mathrm{~N}_{2} \mathrm{O}_{2}$ & 145.0983 & 1.21 \\
\hline \multirow{8}{*}{$\begin{array}{l}\text { negative ion mode of } \\
\text { ESI Amino Acids }\end{array}$} & 8 & $\gamma$-hydroxylysine ${ }^{\mathrm{b}}$ & $\mathrm{C}_{6} \mathrm{H}_{14} \mathrm{~N}_{2} \mathrm{O}_{3}$ & 161.0932 & 1.43 \\
\hline & 9 & Dimethylarginine $^{\text {a }}$ & $\mathrm{C}_{8} \mathrm{H}_{18} \mathrm{~N}_{4} \mathrm{O}_{2}$ & 201.1357 & 1.15 \\
\hline & 10 & L-histidine $^{\text {a }}$ & $\mathrm{C}_{6} \mathrm{H}_{9} \mathrm{~N}_{3} \mathrm{O}_{2}$ & 154.0622 & 1.13 \\
\hline & 11 & O-phospho-ethanolamine a & $\mathrm{C}_{2} \mathrm{H}_{8} \mathrm{NO}_{4} \mathrm{P}$ & 140.0118 & 1.38 \\
\hline & 12 & Taurine $^{\mathrm{a}}$ & $\mathrm{C}_{2} \mathrm{H}_{7} \mathrm{NO}_{3} \mathrm{~S}$ & 124.0074 & 1.37 \\
\hline & 13 & L-citrulline ${ }^{a}$ & $\mathrm{C}_{6} \mathrm{H}_{13} \mathrm{~N}_{3} \mathrm{O}_{3}$ & 174.0884 & 1.34 \\
\hline & 14 & Creatine $^{a}$ & $\mathrm{C}_{4} \mathrm{H}_{9} \mathrm{~N}_{3} \mathrm{O}_{2}$ & 130.0622 & 1.28 \\
\hline & 1 & Linoleic acid ${ }^{\mathrm{b}}$ & $\mathrm{C}_{18} \mathrm{H}_{32} \mathrm{O}_{2}$ & 279.233 & 20.64 \\
\hline \multirow{3}{*}{$\begin{array}{l}\text { Nonolar extracts in } \\
\text { negative ion mode of }\end{array}$} & 2 & palmitoleic acid ${ }^{\mathrm{b}}$ & $\mathrm{C}_{16} \mathrm{H}_{30} \mathrm{O}_{2}$ & 253.2173 & 19.24 \\
\hline & 3 & Oleic acid $^{\mathrm{b}}$ & $\mathrm{C}_{18} \mathrm{H}_{34} \mathrm{O}_{2}$ & 281.2486 & 24.78 \\
\hline & 4 & Arachidonic acid ${ }^{\text {a }}$ & $\mathrm{C}_{20} \mathrm{H}_{32} \mathrm{O}_{2}$ & 303.233 & 19.67 \\
\hline \multirow[t]{2}{*}{ ESI Fatty acids } & 5 & FAC $14: 1-\mathrm{OH}^{\mathrm{b}}$ & $\mathrm{C}_{14} \mathrm{H}_{26} \mathrm{O}_{3}$ & 241.1809 & 7.69 \\
\hline & 6 & Niacinamide $^{\text {a }}$ & $\mathrm{C}_{6} \mathrm{H}_{6} \mathrm{~N}_{2} \mathrm{O}$ & 121.0407 & 5.05 \\
\hline Nonolar extracts in & 1 & Galactose $^{\text {a }}$ & $\mathrm{C}_{12} \mathrm{H}_{22} \mathrm{O}_{11}$ & 341.1089 & 1.42 \\
\hline negative ion mode of & 2 & Melezitose $^{\mathrm{b}}$ & $\mathrm{C}_{18} \mathrm{H}_{32} \mathrm{O}_{16}$ & 503.1618 & 1.43 \\
\hline $\begin{array}{c}\text { ESI Low-level } \\
\text { metabolites }\end{array}$ & 3 & alpha-Tocopherol ${ }^{\mathrm{b}}$ & $\mathrm{C}_{29} \mathrm{H}_{50} \mathrm{O}_{2}$ & 429.3738 & 31.49 \\
\hline
\end{tabular}

${ }^{a}$ Metabolites confirmed using standard compounds. ${ }^{b}$ Metabolites identified by database searches and MS fragmentation. ${ }^{\mathrm{c}}$ As target ions with its exact mass and retention time. 
Table S2. Method sensitivity.

\begin{tabular}{|c|c|c|c|c|c|c|c|c|c|c|c|}
\hline \multirow[b]{3}{*}{ Analytes } & \multirow{3}{*}{$\begin{array}{c}\text { Normal Tissue } \\
\text { Value } \\
\text { (nmol/g) }\end{array}$} & \multicolumn{5}{|c|}{ C18 } & \multicolumn{5}{|c|}{ HILIC } \\
\hline & & \multirow[b]{2}{*}{$\begin{array}{l}\text { RT } \\
(\mathrm{min})\end{array}$} & \multicolumn{2}{|c|}{$(+)$ ESI } & \multicolumn{2}{|c|}{$(-) E S I$} & \multirow[b]{2}{*}{$\begin{array}{c}\text { RT } \\
(\mathrm{min})\end{array}$} & \multicolumn{2}{|c|}{$(+)$ ESI } & \multicolumn{2}{|c|}{$(-)$ ESI } \\
\hline & & & $\begin{array}{l}\text { LOD } \\
\text { (nmol/g) }\end{array}$ & $\mathrm{S} / \mathrm{N}$ & $\begin{array}{l}\text { LOD } \\
\text { (nmol/ } \\
\text { g) }\end{array}$ & $\mathrm{S} / \mathrm{N}$ & & $\begin{array}{l}\text { LOD } \\
\text { (nmol/g) }\end{array}$ & $\mathrm{S} / \mathrm{N}$ & $\begin{array}{l}\text { LOD } \\
\text { (nmol/g) }\end{array}$ & $S / N$ \\
\hline L-Tyrosine & $\begin{array}{c}102.685 \\
(33.22-172.15)\end{array}$ & 1.64 & 10.3 & 2904 & - & - & 10.76 & 10.3 & 8 & - & - \\
\hline Proline & $\begin{array}{c}250.475 \\
(80.54-420.41)\end{array}$ & 1.41 & 25.0 & 4591 & - & - & 12.47 & 25.0 & 72 & - & - \\
\hline Valine & $\begin{array}{c}415.13 \\
(78.54-751.72)\end{array}$ & 1.44 & 41.5 & 1693 & - & - & 11.52 & 41.5 & 31 & - & - \\
\hline Phenylalanine & $\begin{array}{c}142.355 \\
(41.89-242.82)\end{array}$ & 3.73 & 14.2 & 313 & - & - & 11.65 & 14.2 & 21 & - & - \\
\hline Tryptophan & $\begin{array}{c}47.23 \\
(13.17-81.29)\end{array}$ & 4.09 & 4.7 & 1813 & 4.7 & 688 & 10.03 & 4.7 & 13 & 4.7 & 7 \\
\hline Kynurenic acid & 304.998 & 5.08 & 30.5 & 2259 & 30.5 & 4102 & 1.35 & 30.5 & 153 & - & - \\
\hline Arachidonic acid & 304.998 & 19.62 & 30.5 & 220 & 30.5 & 3495 & 1.32 & 30.5 & 223 & 30.5 & 142 \\
\hline Nicotinic acid & 304.998 & 2.09 & 30.5 & 190 & - & - & 5.62 & 30.5 & 169 & - & - \\
\hline Hippuric acid & $\begin{array}{c}5.535 \\
(0.50-10.57)\end{array}$ & 4.78 & 0.6 & 205 & - & - & 4.74 & 0.6 & 10 & - & - \\
\hline Linoleic acid & 304.998 & 20.53 & 30.5 & 54 & 30.5 & 612 & 1.31 & 30.5 & 96 & 30.5 & 112 \\
\hline Oleic acid & 304.998 & 24.80 & 30.5 & 53 & 30.5 & 581 & 1.29 & 30.5 & 314 & 30.5 & 108 \\
\hline L-Carnitine & $\begin{array}{c}604.46 \\
(105.08-1103.8 \\
4)\end{array}$ & 4.05 & 60.4 & 4798 & - & - & 19.04 & 60.4 & 243 & - & - \\
\hline LysoPC 16:0 & 304.998 & 11.38 & 30.5 & 5130 & 30.5 & 74 & 13.30 & 30.5 & 518 & - & - \\
\hline Acetylcarnitine & $\begin{array}{c}143.065 \\
(3.47-282.66)\end{array}$ & 1.30 & 14.3 & 6661 & 14.3 & 49 & 18.21 & 14.3 & 98 & 14.3 & 3213 \\
\hline Octanoylcarnitine & 304.998 & 4.99 & 30.5 & 5140 & 30.5 & 84 & 14.29 & 30.5 & 692 & 30.5 & 475 \\
\hline Decanoylcarnitine & 304.998 & 5.43 & 30.5 & 9171 & 30.5 & 411 & 13.72 & 30.5 & 432 & 30.5 & 2857 \\
\hline Cytidine & $\begin{array}{c}60.74 \\
(5.39-116.09)\end{array}$ & 1.25 & 6.1 & 1169 & 6.1 & 215 & 5.29 & 6.1 & 451 & 6.1 & 148 \\
\hline Inosine & $\begin{array}{c}225.175 \\
(8.58-441.77)\end{array}$ & 3.64 & 22.5 & 132 & 22.5 & 48 & 3.87 & 22.5 & 158 & 22.5 & 11 \\
\hline $\begin{array}{c}\text { Methyltestosteron } \\
\text { e }\end{array}$ & 304.998 & 7.28 & 30.5 & 1531 & 30.5 & 438 & 1.31 & 30.5 & 57 & 30.5 & 136 \\
\hline
\end{tabular}


Table S3. Instrument precision.

\begin{tabular}{|c|c|c|c|c|c|c|c|c|}
\hline \multirow{3}{*}{ Analytes } & \multicolumn{4}{|c|}{ RPLC-MS } & \multicolumn{4}{|c|}{ HILIC-MS } \\
\hline & \multicolumn{2}{|c|}{ Positive ion mode } & \multicolumn{2}{|c|}{ Negative ion mode } & \multicolumn{2}{|c|}{ Positive ion mode } & \multicolumn{2}{|c|}{ Negative ion mode } \\
\hline & $\begin{array}{c}\text { Average } \\
\text { Area } \\
\text { (counts) }\end{array}$ & $\begin{array}{l}\text { RSD } \\
(\%)\end{array}$ & $\begin{array}{c}\text { Average } \\
\text { Area } \\
\text { (counts) }\end{array}$ & $\begin{array}{l}\text { RSD } \\
\text { (\%) }\end{array}$ & $\begin{array}{c}\text { Average } \\
\text { Area } \\
\text { (counts) }\end{array}$ & $\begin{array}{l}\text { RSD } \\
\text { (\%) }\end{array}$ & $\begin{array}{c}\text { Average } \\
\text { Area } \\
\text { (counts) }\end{array}$ & $\begin{array}{l}\text { RSD } \\
\text { (\%) }\end{array}$ \\
\hline L-Tyrosine & $1.07 \mathrm{E}+09$ & 3.7 & - & - & $3.45 \mathrm{E}+09$ & 10.9 & - & - \\
\hline Proline & $8.64 \mathrm{E}+09$ & 5.9 & - & - & $2.51 \mathrm{E}+10$ & 9.4 & - & - \\
\hline Valine & $1.04 \mathrm{E}+10$ & 6.3 & - & - & $1.63 \mathrm{E}+10$ & 12.7 & - & - \\
\hline Phenylalanine & $2.02 E+10$ & 3.2 & - & - & $4.15 \mathrm{E}+10$ & 16.2 & - & - \\
\hline Tryptophan & $4.92 E+09$ & 4.4 & $7.12 E+08$ & 7.7 & $4.21 \mathrm{E}+09$ & 12.6 & $1.05 E+10$ & 11.7 \\
\hline Kynurenic acid & $3.58 \mathrm{E}+10$ & 5.2 & $1.30 \mathrm{E}+10$ & 14.2 & $3.85 E+10$ & 14.9 & - & - \\
\hline Arachidonic acid & $1.66 \mathrm{E}+09$ & 3.6 & $2.65 \mathrm{E}+10$ & 3.8 & $7.65 \mathrm{E}+06$ & 7.6 & $2.29 \mathrm{E}+10$ & 9.5 \\
\hline Nicotinic acid & $5.23 E+10$ & 4.7 & - & - & $7.22 \mathrm{E}+10$ & 2.9 & - & - \\
\hline Hippuric acid & $7.38 \mathrm{E}+08$ & 7.9 & - & - & $3.41 \mathrm{E}+07$ & 7.9 & - & - \\
\hline Linoleic acid & $2.35 \mathrm{E}+08$ & 4.7 & $1.71 \mathrm{E}+10$ & 4.4 & $3.01 \mathrm{E}+06$ & 6.5 & $3.42 \mathrm{E}+10$ & 8.2 \\
\hline Oleic acid & $1.01 \mathrm{E}+11$ & 5.4 & $2.33 E+10$ & 3.4 & $4.33 E+05$ & 8.6 & $3.38 \mathrm{E}+10$ & 8.0 \\
\hline L-Carnitine & $1.02 \mathrm{E}+11$ & 6.2 & - & - & $2.09 \mathrm{E}+11$ & 4.2 & - & - \\
\hline LysoPC 16:0 & $7.89 E+10$ & 3.4 & $5.39 \mathrm{E}+06$ & 5.2 & $9.15 \mathrm{E}+10$ & 7.9 & - & - \\
\hline Acetylcarnitine & $4.02 \mathrm{E}+10$ & 4.7 & $1.44 \mathrm{E}+06$ & 3.3 & $9.40 \mathrm{E}+10$ & 3.6 & $2.36 \mathrm{E}+08$ & 2.5 \\
\hline Octanoylcarnitine & $2.24 \mathrm{E}+11$ & 4.1 & $1.29 E+07$ & 11.4 & $2.72 E+11$ & 9.3 & $1.21 \mathrm{E}+09$ & 3.7 \\
\hline Decanoylcarnitine & $2.39 \mathrm{E}+11$ & 4.3 & $5.02 \mathrm{E}+07$ & 1.9 & $2.23 E+11$ & 6.5 & $9.71 E+08$ & 3.1 \\
\hline Cytidine & $1.16 \mathrm{E}+09$ & 6.2 & $4.54 \mathrm{E}+08$ & 2.9 & $1.77 \mathrm{E}+10$ & 8.7 & $5.63 E+09$ & 2.9 \\
\hline Inosine & $1.19 \mathrm{E}+09$ & 7.1 & $5.91 \mathrm{E}+08$ & 7.3 & $1.54 \mathrm{E}+10$ & 10.2 & $6.35 E+10$ & 2.6 \\
\hline $\begin{array}{c}\text { Methyltestosteron } \\
\text { e }\end{array}$ & $1.32 \mathrm{E}+11$ & 4.1 & $6.91 E+07$ & 3.7 & $7.29 E+09$ & 7.7 & $1.29 E+08$ & 7.9 \\
\hline
\end{tabular}


Table S4. Method precision.

\begin{tabular}{|c|c|c|c|c|c|c|c|c|}
\hline \multirow{3}{*}{ Analytes } & \multicolumn{4}{|c|}{ RPLC-MS } & \multicolumn{4}{|c|}{ HILIC-MS } \\
\hline & \multicolumn{2}{|c|}{$\begin{array}{l}\text { Positive ion mode } \\
\text { RSD(\%) }\end{array}$} & \multicolumn{2}{|c|}{$\begin{array}{l}\text { Negative ion mode } \\
\qquad \text { RSD(\%) }\end{array}$} & \multicolumn{2}{|c|}{$\begin{array}{l}\text { Positive ion mode } \\
\text { RSD(\%) }\end{array}$} & \multicolumn{2}{|c|}{$\begin{array}{c}\text { Negative ion } \\
\text { mode } \\
\text { RSD(\%) }\end{array}$} \\
\hline & $\begin{array}{c}\text { Intra-batc } \\
h \\
(n=6)\end{array}$ & $\begin{array}{c}\text { Inter-batc } \\
\qquad \begin{array}{c}h \\
(n=3)\end{array}\end{array}$ & $\begin{array}{l}\text { Intra- } \\
\text { batch }\end{array}$ & $\begin{array}{l}\text { Inter- } \\
\text { batch }\end{array}$ & $\begin{array}{l}\text { Intra- } \\
\text { batch }\end{array}$ & $\begin{array}{l}\text { Inter- } \\
\text { batch }\end{array}$ & $\begin{array}{l}\text { Intra- } \\
\text { batch }\end{array}$ & $\begin{array}{l}\text { Inter- } \\
\text { batch }\end{array}$ \\
\hline L-Tyrosine & 9.8 & 12.4 & - & - & 10.8 & 12.9 & - & - \\
\hline Proline & 14.6 & 16.2 & - & - & 16.4 & 23.2 & - & - \\
\hline Valine & 12.9 & 14.2 & - & - & 13.0 & 17.0 & - & - \\
\hline Phenylalanine & 12.3 & 12.2 & - & - & 16.6 & 17.9 & - & - \\
\hline Tryptophan & 9.4 & 9.8 & 14.6 & 17.5 & 18.2 & 21.2 & 13.0 & 12.9 \\
\hline Kynurenic acid & 8.6 & 10.7 & 17.9 & 19.8 & 15.0 & 14.7 & & \\
\hline Arachidonic acid & 5.5 & 6.6 & 5.5 & 5.8 & 16.0 & 18.7 & 12.3 & 17.7 \\
\hline Nicotinic acid & 7.0 & 10.2 & - & - & 14.0 & 13.6 & - & - \\
\hline Hippuric acid & 10.1 & 18.2 & - & - & - & - & - & - \\
\hline Linoleic acid & 3.5 & 4.4 & 7.9 & 7.7 & 15.8 & 15.8 & 12.9 & 16.6 \\
\hline Oleic acid & 7.6 & 8.0 & 12.8 & 12.2 & 18.8 & 23.1 & 10.1 & 10.4 \\
\hline L-Carnitine & 14.8 & 17.1 & - & - & 10.8 & 10.5 & - & - \\
\hline LysoPC 16:0 & 5.9 & 8.8 & 10.8 & 13.7 & 14.6 & 14.2 & - & - \\
\hline Acetylcarnitine & 10.4 & 12.1 & 9.3 & 9.4 & 14.6 & 14.1 & 15.7 & 16.0 \\
\hline $\begin{array}{c}\text { Octanoylcarniti } \\
\text { ne }\end{array}$ & 7.5 & 9.2 & 11.1 & 10.8 & 7.0 & 7.2 & 6.8 & 7.5 \\
\hline $\begin{array}{c}\text { Decanoylcarniti } \\
\text { ne }\end{array}$ & 4.9 & 5.8 & 7.0 & 8.5 & 7.8 & 7.3 & 13.0 & 13.2 \\
\hline Cytidine & 12.5 & 15.1 & 20.9 & 20.1 & 14.1 & 14.8 & 8.6 & 9.3 \\
\hline Inosine & 10.9 & 12.0 & - & - & 11.1 & 11.2 & 8.8 & 10.1 \\
\hline $\begin{array}{l}\text { Methyltestoster } \\
\text { one }\end{array}$ & 3.7 & 4.5 & 9.5 & 9.4 & 12.7 & 12.7 & 10.7 & 12.0 \\
\hline
\end{tabular}


Table S5. Extraction recoveries of endogenous metabolites.

\begin{tabular}{|c|c|c|c|c|c|}
\hline \multirow{2}{*}{ Analytes } & \multirow{2}{*}{ Spiked level } & \multirow{2}{*}{$\begin{array}{c}\text { Mean area of } \\
\text { RPLC-MS } \\
\text { (sample/reference) }\end{array}$} & \multirow{2}{*}{$\begin{array}{c}\text { Mean area of } \\
\text { HILIC-MS } \\
\text { (sample/reference) }\end{array}$} & \multicolumn{2}{|c|}{ Recovery rate } \\
\hline & & & & (C18/HILIC) & SUM \\
\hline \multirow{4}{*}{ L-Tyrosine } & blank tissue & 8.27E+06 & $4.38 \mathrm{E}+07$ & - & - \\
\hline & low & $2.97 E+07 / 6.38 E+08$ & $4.95 E+07 / 5.37 E+07$ & $3.4 / 57.5$ & 60.9 \\
\hline & middle & $3.35 \mathrm{E}+07 / 8.90 \mathrm{E}+08$ & $7.41 E+08 / 8.74 E+08$ & $2.9 / 84.0$ & 86.9 \\
\hline & high & $9.38 E+07 / 1.03 E+09$ & $4.46 \mathrm{E}+08 / 6.94 \mathrm{E}+08$ & $8.4 / 61.8$ & 70.2 \\
\hline \multirow{4}{*}{ Proline } & blank tissue & $3.24 \mathrm{E}+07$ & $1.39 \mathrm{E}+09$ & - & - \\
\hline & low & $1.11 E+08 / 3.07 E+09$ & $2.53 E+09 / 2.62 E+09$ & $2.6 / 92.4$ & 95.0 \\
\hline & middle & $2.00 E+08 / 4.63 E+09$ & $1.06 E+10 / 1.23 E+10$ & $3.6 / 84.1$ & 87.7 \\
\hline & high & $5.13 E+08 / 8.53 E+09$ & $9.57 E+09 / 1.37 E+10$ & $5.7 / 66.6$ & 72.3 \\
\hline \multirow{4}{*}{ Valine } & blank tissue & $3.85 E+07$ & $4.21 E+09$ & - & - \\
\hline & low & $1.81 E+08 / 2.16 \mathrm{E}+09$ & $4.66 \mathrm{E}+09 / 1.06 \mathrm{E}+10$ & $6.7 / 44.0$ & 50.7 \\
\hline & middle & $4.25 E+08 / 3.32 E+09$ & $1.02 E+10 / 1.01 E+10$ & $11.8 / 101.5$ & 113.3 \\
\hline & high & $7.28 \mathrm{E}+08 / 5.84 \mathrm{E}+09$ & $7.91 \mathrm{E}+09 / 8.38 \mathrm{E}+09$ & $11.9 / 88.8$ & 100.7 \\
\hline \multirow{4}{*}{ Phenylalanine } & blank tissue & $6.39 E+06$ & $5.64 E+08$ & - & - \\
\hline & low & $2.47 \mathrm{E}+07 / 6.80 \mathrm{E}+08$ & $7.77 E+08 / 8.05 E+08$ & $2.7 / 88.3$ & 91.0 \\
\hline & middle & $3.12 E+07 / 1.29 E+09$ & $5.21 E+09 / 5.95 E+09$ & $1.9 / 86.2$ & 88.1 \\
\hline & high & $8.16 E+07 / 2.55 E+09$ & $5.25 E+09 / 5.65 E+09$ & $3.0 / 92.2$ & 95.2 \\
\hline \multirow{4}{*}{ Tryptophan } & blank tissue & $9.87 E+05$ & $2.50 \mathrm{E}+07$ & - & - \\
\hline & low & $3.45 E+06 / 1.23 E+08$ & $2.60 \mathrm{E}+07 / 2.62 \mathrm{E}+07$ & $2.0 / 81.4$ & 83.4 \\
\hline & middle & $4.49 E+06 / 2.51 E+08$ & $3.30 \mathrm{E}+08 / 4.79 \mathrm{E}+08$ & $1.4 / 67.1$ & 68.5 \\
\hline & high & $1.23 \mathrm{E}+07 / 5.45 \mathrm{E}+08$ & $3.29 E+08 / 4.93 E+08$ & $2.1 / 65.0$ & 67.1 \\
\hline \multirow{4}{*}{ Kynurenic acid } & blank tissue & $1.71 \mathrm{E}+07$ & $4.06 \mathrm{E}+07$ & - & - \\
\hline & low & $2.10 E+08 / 1.61 E+10$ & $4.64 \mathrm{E}+09 / 6.12 \mathrm{E}+09$ & $1.2 / 75.6$ & 76.8 \\
\hline & middle & $3.57 E+08 / 2.35 E+10$ & $8.77 E+09 / 7.89 E+09$ & $1.5 / 111.2$ & 112.7 \\
\hline & high & $1.88 \mathrm{E}+09 / 3.58 \mathrm{E}+10$ & $9.72 E+09 / 1.15 E+10$ & $5.2 / 84.8$ & 90.0 \\
\hline \multirow{4}{*}{ Arachidonic acid } & blank tissue & $1.33 \mathrm{E}+08$ & $7.79 E+05$ & - & - \\
\hline & low & $4.51 E+08 / 4.20 E+08$ & $8.75 E+05 / 3.39 E+06$ & $110.8 / 3.7$ & 114.5 \\
\hline & middle & $4.67 \mathrm{E}+08 / 4.75 \mathrm{E}+08$ & $1.30 \mathrm{E}+06 / 3.58 \mathrm{E}+06$ & $97.6 / 18.6$ & 116.2 \\
\hline & high & $7.87 E+08 / 8.24 E+08$ & $1.75 E+06 / 5.81 E+06$ & $94.6 / 19.3$ & 113.9 \\
\hline \multirow{4}{*}{ Nicotinic acid } & blank tissue & $1.31 \mathrm{E}+07$ & $7.63 E+08$ & - & - \\
\hline & low & $2.64 E+07 / 9.95 E+09$ & $8.44 E+09 / 1.37 E+10$ & $4.2 / 59.4$ & 63.6 \\
\hline & middle & $3.61 E+07 / 1.62 E+10$ & $2.60 E+10 / 3.05 E+10$ & $3.8 / 85.1$ & 88.9 \\
\hline & high & $7.45 \mathrm{E}+07 / 2.87 \mathrm{E}+10$ & $2.96 E+10 / 4.33 E+10$ & $5.3 / 67.8$ & 73.1 \\
\hline \multirow{4}{*}{ Hippuric acid } & blank tissue & $3.76 \mathrm{E}+06$ & - & - & - \\
\hline & low & $9.21 E+06 / 7.86 \mathrm{E}+07$ & - & $7.3 /-$ & 7.3 \\
\hline & middle & $1.49 E+07 / 1.45 E+08$ & - & $7.9 /-$ & 7.9 \\
\hline & high & $2.56 \mathrm{E}+07 / 3.22 \mathrm{E}+08$ & - & $6.9 /-$ & 6.9 \\
\hline \multirow{4}{*}{ Linoleic acid } & blank tissue & $1.31 \mathrm{E}+07$ & $2.33 E+05$ & - & - \\
\hline & low & $4.71 \mathrm{E}+07 / 4.81 \mathrm{E}+07$ & $1.01 \mathrm{E}+06 / 2.71 \mathrm{E}+06$ & $97.3 / 31.3$ & 128.6 \\
\hline & middle & $3.61 \mathrm{E}+07 / 5.65 \mathrm{E}+07$ & $1.22 \mathrm{E}+06 / 4.12 \mathrm{E}+06$ & $53.1 / 25.5$ & 78.6 \\
\hline & high & $7.45 E+07 / 9.54 E+07$ & $5.85 E+05 / 4.67 E+06$ & $74.7 / 8.0$ & 82.7 \\
\hline
\end{tabular}


Continued

\begin{tabular}{|c|c|c|c|c|c|}
\hline \multirow[t]{2}{*}{ Analytes } & \multirow[t]{2}{*}{ Spiked level } & \multirow{2}{*}{$\begin{array}{c}\text { Mean area of } \\
\text { RPLC-MS } \\
\text { (sample/reference) }\end{array}$} & \multirow{2}{*}{$\begin{array}{c}\text { Mean area of } \\
\text { HILIC-MS } \\
\text { (sample/reference) }\end{array}$} & \multicolumn{2}{|c|}{ Recovery rate } \\
\hline & & & & (C18/HILIC) & SUM \\
\hline \multirow{4}{*}{ Oleic acid } & blank tissue & $3.76 \mathrm{E}+06$ & $9.53 E+04$ & - & - \\
\hline & low & $1.61 \mathrm{E}+07 / 1.52 \mathrm{E}+07$ & $9.83 E+04 / 1.13 E+05$ & $108.3 / 17.6$ & 125.9 \\
\hline & middle & $1.49 \mathrm{E}+07 / 1.71 \mathrm{E}+07$ & $1.19 E+05 / 1.73 E+05$ & $83.5 / 30.3$ & 113.8 \\
\hline & high & $2.56 \mathrm{E}+07 / 3.11 \mathrm{E}+07$ & $1.22 \mathrm{E}+05 / 1.60 \mathrm{E}+05$ & $79.8 / 41.6$ & 121.4 \\
\hline \multirow{4}{*}{ L-Carnitine } & blank tissue & $2.30 \mathrm{E}+08$ & $2.10 \mathrm{E}+10$ & - & - \\
\hline & low & $7.12 E+08 / 1.91 E+10$ & $3.73 E+10 / 3.99 E+10$ & $2.6 / 86.4$ & 89.0 \\
\hline & middle & $8.78 E+08 / 3.10 E+10$ & $8.84 E+10 / 1.01 E+11$ & $2.1 / 84.0$ & 86.1 \\
\hline & high & $2.80 E+09 / 5.64 E+10$ & $9.76 E+10 / 1.35 E+11$ & $4.6 / 67.4$ & 72.0 \\
\hline \multirow{4}{*}{ LysoPC 16:0 } & blank tissue & $1.49 \mathrm{E}+09$ & $5.31 E+08$ & - & - \\
\hline & low & $6.25 E+09 / 7.86 E+09$ & $1.28 \mathrm{E}+09 / 5.08 \mathrm{E}+09$ & $74.7 / 16.6$ & 91.3 \\
\hline & middle & $9.34 \mathrm{E}+09 / 1.22 \mathrm{E}+10$ & $1.49 \mathrm{E}+09 / 9.26 \mathrm{E}+09$ & $73.4 / 11.0$ & 84.4 \\
\hline & high & $1.67 \mathrm{E}+10 / 2.24 \mathrm{E}+10$ & $9.76 E+10 / 1.90 E+10$ & $72.7 / 15.4$ & 88.1 \\
\hline \multirow{4}{*}{ Acetylcarnitine } & blank tissue & $6.60 E+07$ & $8.16 E+09$ & - & - \\
\hline & low & $2.98 \mathrm{E}+08 / 7.14 \mathrm{E}+09$ & $1.27 \mathrm{E}+10 / 1.27 \mathrm{E}+10$ & 3.3/99.9 & 103.2 \\
\hline & middle & $3.63 E+08 / 1.15 E+10$ & $3.94 E+10 / 4.54 E+10$ & $2.6 / 84.1$ & 86.7 \\
\hline & high & $1.26 \mathrm{E}+09 / 2.16 \mathrm{E}+10$ & $3.91 \mathrm{E}+10 / 6.12 \mathrm{E}+10$ & $5.6 / 58.4$ & 64.0 \\
\hline \multirow{4}{*}{ Octanoylcarnitine } & blank tissue & $6.70 E+07$ & $2.66 \mathrm{E}+08$ & - & - \\
\hline & low & $5.80 E+09 / 5.00 E+10$ & $1.76 \mathrm{E}+10 / 2.78 \mathrm{E}+10$ & $11.5 / 63.0$ & 74.5 \\
\hline & middle & $1.06 E+10 / 7.23 E+10$ & $5.90 E+10 / 6.38 E+10$ & $14.5 / 92.4$ & 106.9 \\
\hline & high & $2.49 E+10 / 1.14 E+11$ & $7.74 E+10 / 1.15 E+11$ & $21.8 / 67.1$ & 88.9 \\
\hline \multirow{4}{*}{ Decanoylcarnitine } & blank tissue & $5.81 E+07$ & $2.89 E+08$ & - & - \\
\hline & low & $1.39 E+10 / 4.80 E+10$ & $1.53 E+10 / 3.11 E+10$ & $28.8 / 48.5$ & 77.3 \\
\hline & middle & $2.43 E+10 / 7.09 E+10$ & $1.85 E+10 / 4.70 E+10$ & $34.3 / 39.0$ & 73.3 \\
\hline & high & $5.01 E+10 / 1.13 E+11$ & $5.71 E+10 / 7.57 E+10$ & $44.4 / 75.3$ & 119.7 \\
\hline \multirow{4}{*}{ Cytidine } & blank tissue & $5.33 E+05$ & $6.38 \mathrm{E}+08$ & - & - \\
\hline & low & $1.56 \mathrm{E}+06 / 1.32 \mathrm{E}+08$ & $8.88 \mathrm{E}+08 / 9.51 \mathrm{E}+08$ & $0.8 / 79.8$ & 80.6 \\
\hline & middle & $2.41 E+06 / 2.27 E+08$ & $3.86 E+09 / 3.95 E+09$ & $0.8 / 97.3$ & 98.1 \\
\hline & high & $1.24 \mathrm{E}+07 / 4.31 \mathrm{E}+08$ & $4.49 E+09 / 6.82 E+09$ & $2.8 / 62.3$ & 65.1 \\
\hline \multirow{4}{*}{ Inosine } & blank tissue & $4.07 E+06$ & $2.43 E+09$ & - & - \\
\hline & low & $1.58 \mathrm{E}+07 / 2.16 \mathrm{E}+08$ & $3.95 E+09 / 4.63 E+09$ & $5.6 / 69.0$ & 74.6 \\
\hline & middle & $1.52 \mathrm{E}+07 / 3.74 \mathrm{E}+08$ & $8.24 E+09 / 8.75 E+09$ & $3.0 / 92.0$ & 95.0 \\
\hline & high & $4.88 \mathrm{E}+07 / 7.78 \mathrm{E}+08$ & $6.68 E+09 / 9.03 E+09$ & $5.8 / 64.3$ & 70.1 \\
\hline \multirow{4}{*}{ Methyltestosterone } & blank tissue & $5.30 E+07$ & $1.49 E+07$ & - & - \\
\hline & low & $3.53 E+10 / 2.39 E+10$ & $1.05 E+08 / 2.31 E+09$ & $114.1 / 3.9$ & 118.0 \\
\hline & middle & $3.51 \mathrm{E}+10 / 3.78 \mathrm{E}+10$ & $1.23 E+08 / 2.81 E+09$ & $92.9 / 3.9$ & 96.8 \\
\hline & high & $7.40 \mathrm{E}+10 / 6.54 \mathrm{E}+10$ & $2.17 E+08 / 2.90 E+09$ & $113.0 / 7.0$ & 120.0 \\
\hline
\end{tabular}


Table S6. Freeze-thaw stability.

\begin{tabular}{|c|c|c|c|c|c|c|c|c|}
\hline \multirow[b]{3}{*}{ Analytes } & \multicolumn{4}{|c|}{ RPLC-MS } & \multicolumn{4}{|c|}{ HILIC-MS } \\
\hline & \multicolumn{2}{|c|}{ Positive ion mode } & \multicolumn{2}{|c|}{ Negative ion mode } & \multicolumn{2}{|c|}{ Positive ion mode } & \multicolumn{2}{|c|}{ Negative ion mode } \\
\hline & $\begin{array}{c}\text { Average } \\
\text { area of } 3 \\
\text { cycles } \\
\text { (counts) }\end{array}$ & $\mathrm{RSD}(\%)$ & $\begin{array}{c}\text { Average } \\
\text { area of } 3 \\
\text { cycles } \\
\text { (counts) }\end{array}$ & $\mathrm{RSD}(\%)$ & $\begin{array}{c}\text { Average } \\
\text { area of } 3 \\
\text { cycles } \\
\text { (counts) }\end{array}$ & $\begin{array}{l}\text { RSD } \\
(\%)\end{array}$ & $\begin{array}{c}\text { Average } \\
\text { area of } 3 \\
\text { cycles } \\
\text { (counts) }\end{array}$ & $\begin{array}{l}\text { RSD } \\
(\%)\end{array}$ \\
\hline L-Tyrosine & $4.19 \mathrm{E}+07$ & 12.1 & - & - & $1.71 \mathrm{E}+08$ & 14.6 & - & - \\
\hline Proline & $2.53 E+08$ & 7.1 & - & - & $9.25 E+09$ & 6.0 & - & - \\
\hline Valine & $1.67 \mathrm{E}+08$ & 9.8 & - & - & $3.52 E+09$ & 3.7 & - & - \\
\hline Phenylalanine & $5.44 \mathrm{E}+07$ & 9.4 & - & - & $2.34 \mathrm{E}+09$ & 1.9 & - & - \\
\hline Tryptophan & $8.27 \mathrm{E}+06$ & 16.9 & $1.31 \mathrm{E}+06$ & 21.5 & $3.49 \mathrm{E}+07$ & 13.7 & $2.64 \mathrm{E}+07$ & 23.2 \\
\hline Kynurenic acid & $8.13 E+08$ & 9.6 & $7.48 \mathrm{E}+07$ & 22.4 & $1.33 \mathrm{E}+10$ & 13.1 & - & - \\
\hline Arachidonic acid & $1.12 \mathrm{E}+09$ & 8.3 & $1.08 \mathrm{E}+10$ & 4.6 & $5.32 \mathrm{E}+05$ & 12.2 & $1.33 \mathrm{E}+07$ & 21.8 \\
\hline Nicotinic acid & $1.65 \mathrm{E}+09$ & 11.6 & - & - & $2.87 E+10$ & 14.3 & - & - \\
\hline Hippuric acid & $3.49 E+06$ & 27.1 & - & - & - & - & - & - \\
\hline Linoleic acid & $1.17 \mathrm{E}+08$ & 11.0 & $3.62 E+09$ & 3.4 & $5.12 \mathrm{E}+05$ & 13.5 & $1.10 \mathrm{E}+08$ & 7.2 \\
\hline Oleic acid & $3.22 \mathrm{E}+07$ & 10.2 & $1.68 \mathrm{E}+09$ & 13.6 & $5.20 \mathrm{E}+04$ & 14.1 & $1.10 \mathrm{E}+08$ & 2.6 \\
\hline L-Carnitine & $1.52 E+09$ & 15.0 & - & - & $8.40 E+10$ & 12.4 & - & - \\
\hline LysoPC 16:0 & $1.34 \mathrm{E}+10$ & 23.6 & $1.09 \mathrm{E}+06$ & 20.8 & $4.56 \mathrm{E}+09$ & 4.8 & - & - \\
\hline Acetylcarnitine & $6.20 E+08$ & 18.4 & $1.60 \mathrm{E}+06$ & 12.0 & $3.19 E+10$ & 12.5 & $2.51 E+07$ & 18.1 \\
\hline Octanoylcarnitine & $1.72 E+10$ & 18.7 & $3.09 E+06$ & 15.4 & $4.85 \mathrm{E}+10$ & 13.8 & $4.40 E+07$ & 13.0 \\
\hline Decanoylcarnitine & $3.83 E+10$ & 21.2 & $8.03 E+06$ & 12.0 & $3.78 \mathrm{E}+10$ & 7.4 & $7.06 \mathrm{E}+07$ & 16.8 \\
\hline Cytidine & $4.86 \mathrm{E}+06$ & 14.9 & $3.26 \mathrm{E}+06$ & 9.6 & $2.66 \mathrm{E}+09$ & 8.9 & $8.74 \mathrm{E}+08$ & 6.3 \\
\hline Inosine & $1.59 \mathrm{E}+07$ & 21.9 & - & - & $4.27 E+09$ & 8.2 & $3.63 \mathrm{E}+10$ & 8.2 \\
\hline $\begin{array}{c}\text { Methyltestosteron } \\
\mathrm{e}\end{array}$ & $9.65 \mathrm{E}+10$ & 9.2 & $1.59 \mathrm{E}+08$ & 5.8 & $3.92 E+08$ & 2.1 & $3.12 \mathrm{E}+06$ & 11.6 \\
\hline
\end{tabular}

Tissue stability after three freeze-thaw cycles $\left(-20^{\circ} \mathrm{C}-20^{\circ} \mathrm{C}\right)$. 


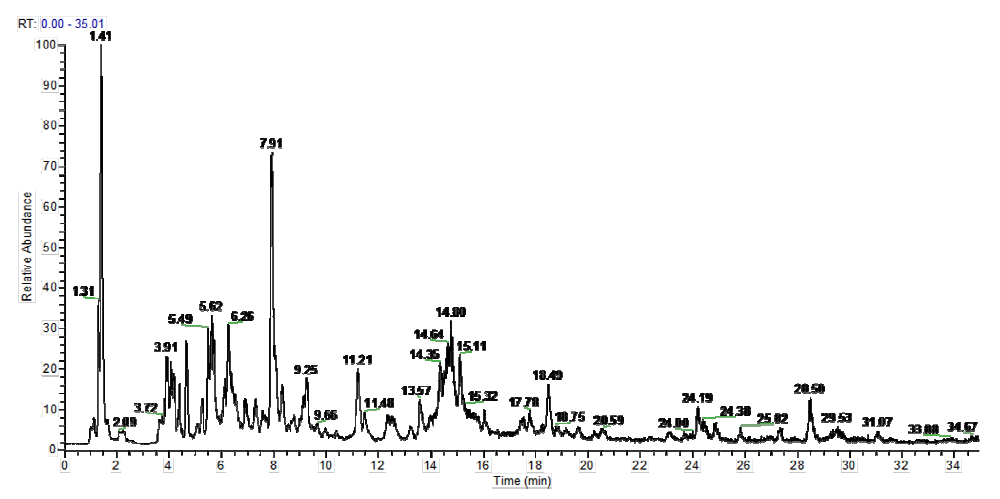

A

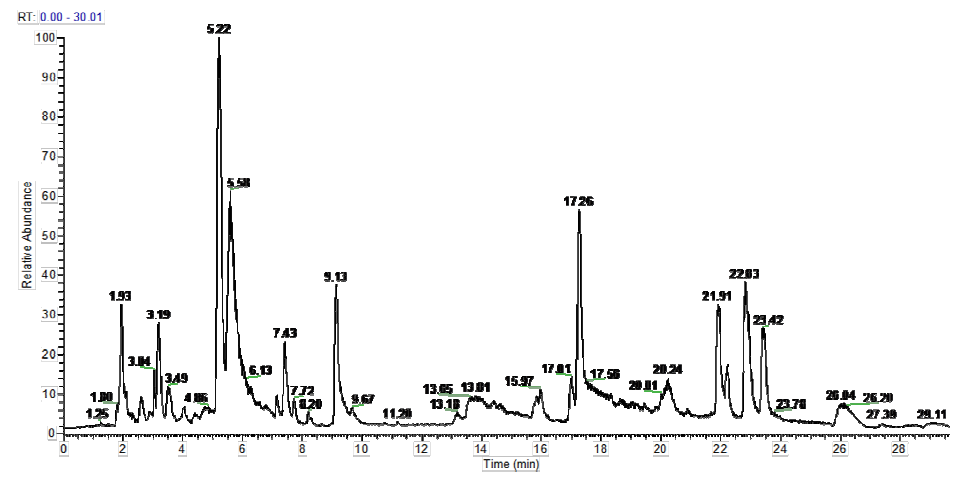

B

Figure S1. Typical TICs obtained from esophagus tissue by LC-(+)ESI-MS. A: organic extracts; B: aqueous extracts.

\section{Nonpolar extracts}

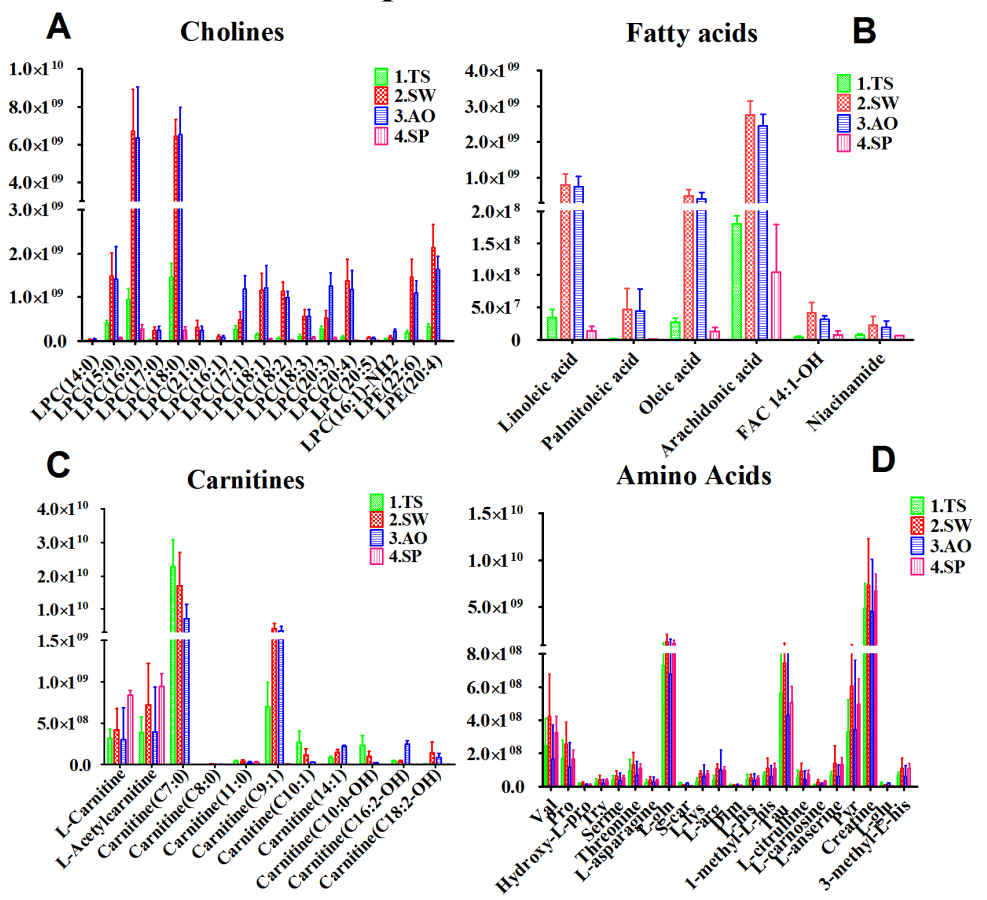

Figure S2. Comparison of extraction efficiency using different solvent extraction methods for cholines (A), fatty acids (B), carnitines (C), and amino acids (D) in nonpolar extracts. Data are shown as column charts. 




Figure S3. Comparison of extraction efficiency using different solvent extraction methods for amino acids (A), nucleosides (B), carnitines (C), and cholines (D) in polar extracts. Data are shown as column charts.

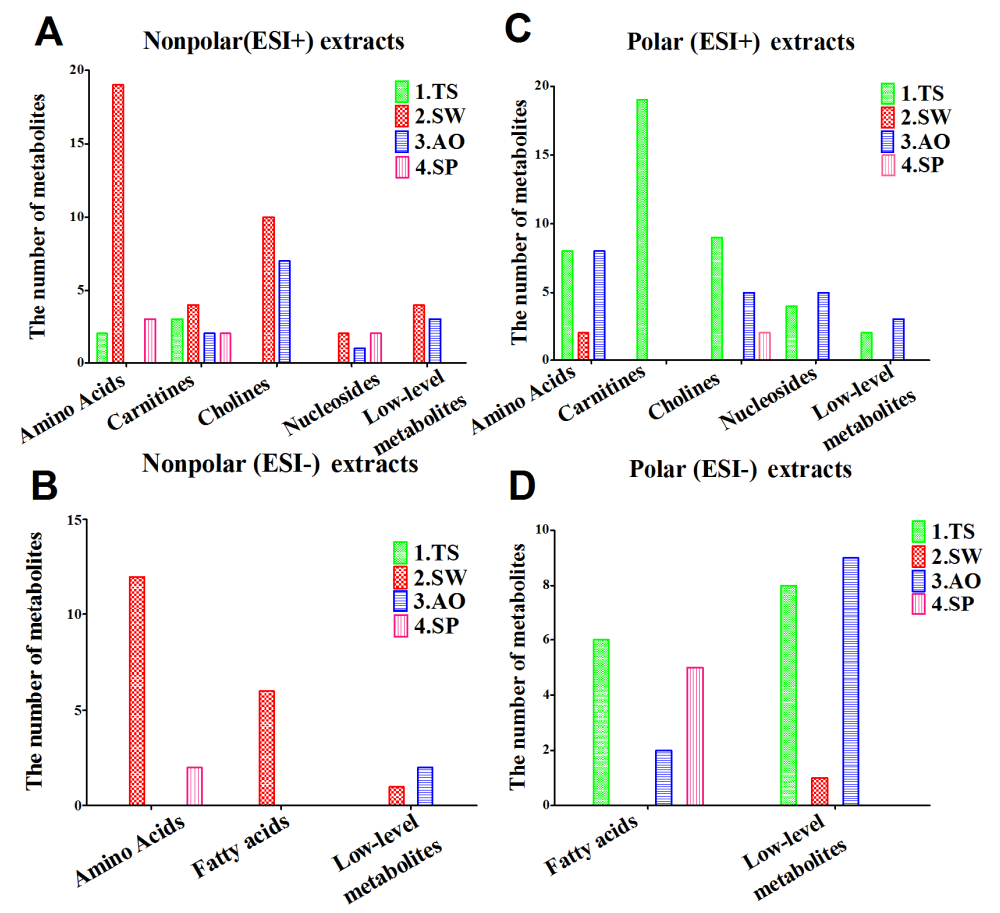

Figure S4. Comparison of extraction efficiency using different solvent extraction methods for positive and negative ion modes of ESI in nonpolar extracts (A, B) and polar extracts (C, D). 

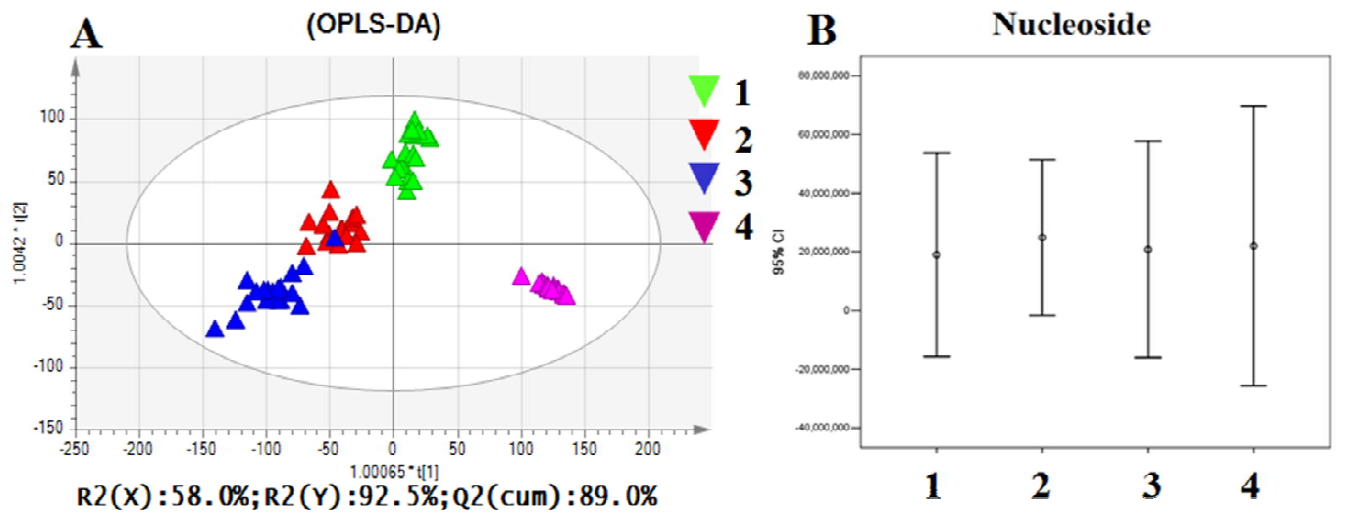

Figure S5. Comparison of method reproducibility, evaluated by OPLS-DA (A) and error bars (B), for positive ion mode of ESI in nonpolar extracts.
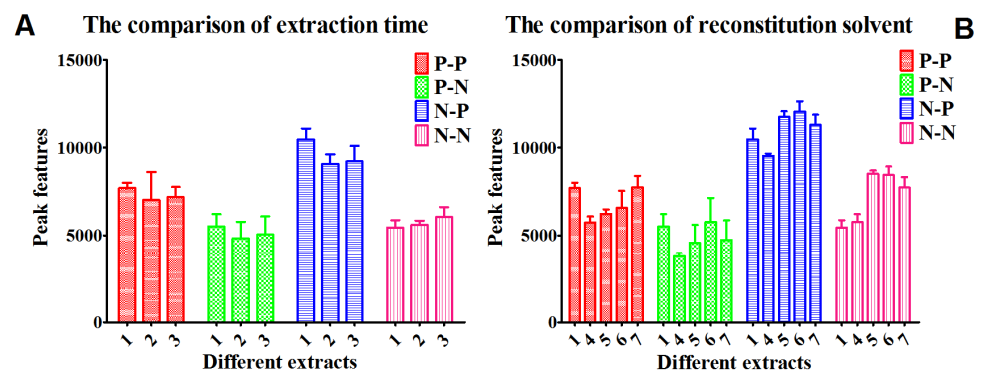

C The ratio comparison of extraction solvent The volume comparison of extraction solvent D

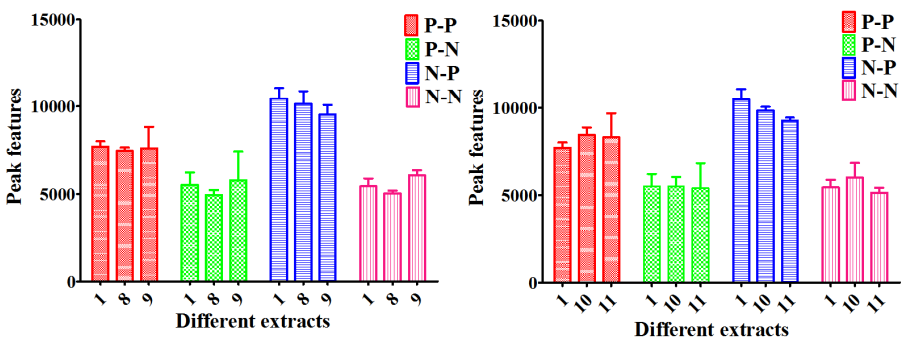

Figure S6. Peak features under different parameters in different extracts. P-P: polar extracts in positive ESI mode; P-N: polar extracts in negative ESI mode; N-P: nonpolar extracts in positive ESI mode; N-N: nonpolar extracts in negative ESI mode. The comparison of extraction time (A): 1 (20min), 2 (40min), 3 (60min). The comparison of reconstitution solvent (B): polar extracts (4: 50/50 acetonitrile/water; 5: 60/40 acetonitrile/water; 6: 80/20 acetonitrile/water; 7: 95/5 acetonitrile/water); nonpolar extracts (4: acetonitrile; 5: 70/30 acetonitrile/water; 6: 40/60 acetonitrile/water; 7: 2/98 acetonitrile/water). The ratio comparison of extraction solvent (C): 1: methanol/water/dichloromethane $(2: 1.5: 2) ; \quad \mathbf{8}$ : methanol/water/dichloromethane $(2: 1: 2) ; \mathbf{9}$ : methanol/water/dichloromethane (1.5:1.5:1). The volume comparison of extraction solvent (D): 1: $1.6 \mathrm{~mL} ; 10: 1.0 \mathrm{~mL} ; 11: 2.0 \mathrm{~mL}$. The parameters we finally chose were $1,6,9$ and 10 after the systematic comparison of polar and nonpolar extracts. 


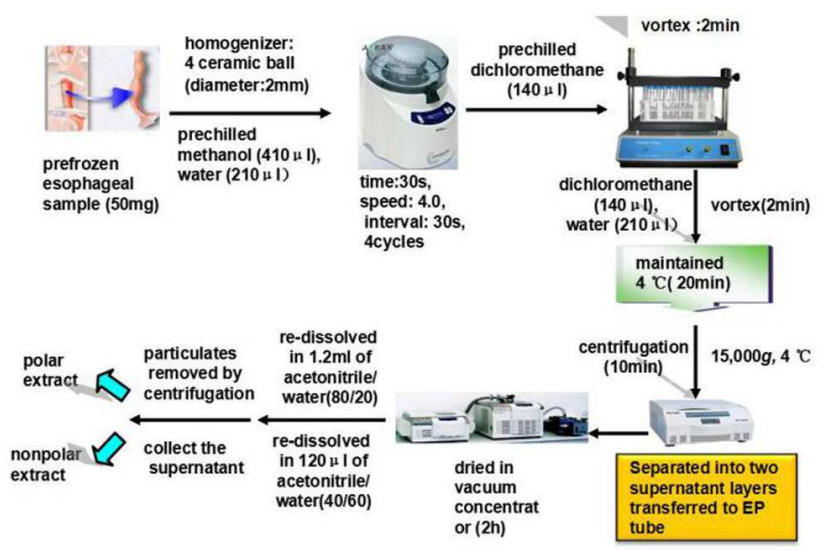

Figure S7. Workflow for esophageal tissue preparation.

\section{SUPPLEMENTARY REFERENCES}

(1) Sugimoto, M.; Ikeda, S.; Niigata, K.; Tomita, M.; Sato, H.; Soga, T. Nucleic. Acids. Res. 2012, 40(Database issue), D809-D814. 